\title{
Spatial Resonant Control of Flexible Structures-Application to a Piezoelectric Laminate Beam
}

\author{
Dunant Halim and S. O. Reza Moheimani, Member, IEEE
}

\begin{abstract}
This paper introduces a class of resonant controllers that can be used to minimize structural vibration using collocated piezoelectric actuator-sensor pairs. The proposed controller increases the damping of the structure so as to minimize a chosen number of resonant responses. The controller can be tuned to a chosen number of modes. This results in controllers of minimal dimension. The controller structure is chosen such that closed-loop stability is guaranteed. Moreover, the controller can be designed such that the spatial $\mathcal{H}_{2}$ norm of the system is minimized. This will guarantee average reduction of vibration throughout the entire structure. Experimental validation on a simply supported beam is presented showing the effectiveness of the proposed controller.
\end{abstract}

Index Terms-Flexible structures, piezoelectric actuator, piezoelectric sensor, smart structures, spatial $\mathcal{H}_{2}$ norm, spatial resonant control, vibrations.

\section{INTRODUCTION}

$\mathbf{E}$ XAMPLES of flexible structures can be found in many systems including aircraft, bridges, and buildings. In reality, however, all structures can be regarded as flexible since they experience structural deformation under some loading, no matter how small. Structures experience vibrations, whose effects are more significant for less rigid structures. In many situations, it is important to be able to minimize these structural vibrations, as they may affect the stability and performance of the structures.

It is well known that it is possible to design controllers which effectively minimize structural vibrations. It is known that the response of flexible structures are relatively large at, or close to, their resonant frequencies. Therefore, it is desirable to have controllers that minimize structural responses at those resonant frequencies. The controller proposed in this paper is motivated by resonant controllers originally developed in [1]. We introduce a more general class of controllers that allows for control of multivariable resonant systems and includes the work of [1] as a special case. The controller is chosen to minimize vibration by increasing the system damping properties for individual modes. The advantage of this particular controller structure is that, in many noise and vibration control problems, it may not

Manuscript received September 5, 1999; revised July 1, 2000 and September 5, 2000. Recommended by Guest Editor G. C. Goodwin. This work was supported by the Australian Research Council and the Centre for Integrated Dynamics and Control.

The authors are with the Department of Electrical and Computer Engineering, University of Newcastle, NSW, Australia (e-mail: dunanth@ee.newcastle.edu.au; reza@ee.newcastle.edu.au).

Publisher Item Identifier S 1063-6536(01)00425-0. be necessary to suppress structural vibration due to every single mode. This is an interesting property which results in controllers of dimensions, not more than necessary.

The proposed controller is applied to a piezoelectric laminate beam. Piezoelectricity was discovered in 1880 by French scientists Pierre and Paul-Jacques Curie. They observed that when they compressed certain types of crystals including quartz, tourmaline, and Rochelle salt, along certain axes, a voltage was produced on the surface of the crystal. In the following year, they discovered the converse effect, namely that such crystals elongated upon the application of an electric current. The piezoelectric effect in natural crystals is rather weak. Therefore, there are limitations in employing them as actuators and sensors for vibration control purposes. Recently, there has been remarkable progress in this field of materials science, which inter-alia has resulted in availability of inexpensive piezoelectric materials. These materials are capable of transforming mechanical energy into electrical energy and vice versa in an efficient way.

There are a number of piezoelectric materials that are currently being used. These include poly-vinylidene flouride (PVDF), which is a semicrystalline polymer film and lead zirconate titanate (PZT), which is a piezoelectric ceramic material. These materials strain when exposed to a voltage and, conversely, produce a voltage when strained. This is due to the permanent dipole nature of these materials. For a detailed discussion of electromechanical properties of these materials the reader is referred to [2]-[4]. When used for vibration control purposes, piezoelectric patches are bonded to the surface or are manufactured into the flexible structure membrane. These patches can then be used as actuators and sensors. An interesting property of piezoelectric actuators and sensors is that they are spatially distributed over the surface which is being sensed or controlled. This property distinguishes them from discrete actuators and sensors that are often used in control of flexible structures. The use of these piezoelectric actuators and sensors have shown promising applications in active vibration control of flexible structures [5]-[10].

An important issue in designing a controller for a flexible structure is whether the developed closed-loop system will have sufficient robustness to deal with uncertainties in the system model. Moreover, transfer functions of flexible structures consist of a large number of highly resonant modes. Controllers are often designed for a limited number of modes that lie within a particular bandwidth and thus these controllers have to guarantee stability in the presence of uncontrolled modes. A controller that is designed for in-bandwidth modes, may result 
in instabilities once implemented on the real systems. This problem is referred to as the spill-over effect [11], [12]. The controller that is proposed in this paper guarantees closed-loop stability in the presence of uncontrolled modes. Moreover, it maintains closed-loop stability even if the resonant frequencies of the system are changed.

The controller is designed such that the spatial $\mathcal{H}_{2}$ norm of the closed-loop system is minimized. The concept of spatial $\mathcal{H}_{2}$ norm was introduced in [5], [13]. Minimizing the spatial $\mathcal{H}_{2}$ norm of the system will guarantee vibration reduction over the entire structure in an average sense. Flexible structures are distributed parameter systems. Therefore, vibration of each point is dynamically related to the vibration of every other point over the structure. If a controller is designed with a view to minimizing structural vibration at a limited number of points, it could have negative effects on the vibration profile for the rest of the structure.

This paper is organized as follows. Section II describes the dynamics of flexible beam structures such as those with collocated piezoelectric actuator-sensor pairs. This section is of a tutorial nature and is included as a ready reference source for researchers entering this field. Section III briefly describes the notion of spatial $\mathcal{H}_{2}$ norm and its use as a performance measure for flexible structures. Section IV presents a controller structure which guarantees closed-loop stability. In Section V, we develop a spatial $\mathcal{H}_{2}$ norm optimization procedure using the Lagrange multiplier technique to optimize over the controller parameters. Section VI discusses the implementation of our single input-single output (SISO) resonant controller to a piezoelectric laminate beam with simply supported ends. Section VII presents experimental validation of the SISO resonant controller applied to the piezoelectric laminate beam. The last section draws conclusions.

\section{Models of Flexible Beam Structures}

In this section, we will develop a model for a piezolectric laminate beam with a number of collocated actuator-sensor pairs using a modal analysis technique. This section has a tutorial nature. The material is available elsewhere in the literature. However, we believe that there is value summarizing it here as a ready reference source for others.

\section{A. Dynamics of a Piezoelectric Laminate Beam}

Consider a flexible structure with a number of piezoelectric actuator-sensor pairs attached to it as shown in Fig. 1. Suppose there are $\bar{I}$ collocated actuator-sensor pairs distributed along the structure. Piezoelectric patches on one side of the beam are used as sensors, while patches on the other side serve as actuators. Voltages that are applied to actuating patches are represented by $V_{a}(t)=\left[V_{a 1}(t), \ldots, V_{a \bar{I}}(t)\right]^{T}$.

We assume that a model of the structure is obtained via the modal analysis procedure. This procedure requires one to find a solution to the partial differential equation (PDE) which describes the dynamics of the flexible structure. The partial differential equation can be solved independently for each mode by using the orthogonality properties of its eigenfunctions, $W_{k}$. Such models have the interesting property that they describe

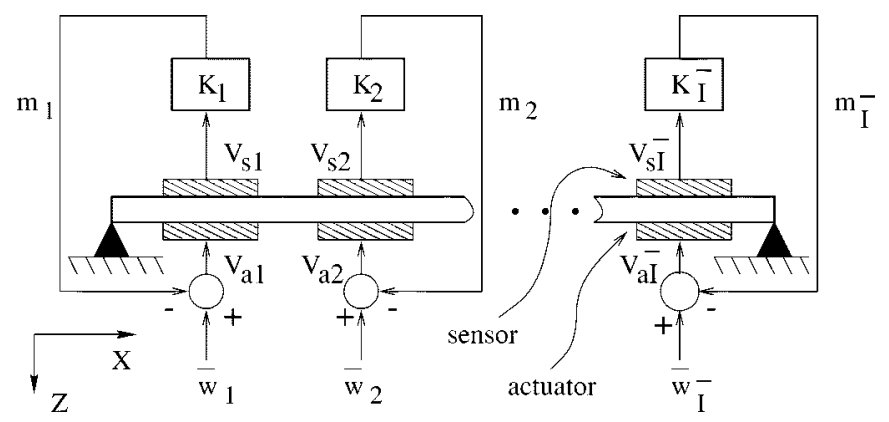

Fig. 1. A simply supported beam with a number of collocated piezoelectric patches.

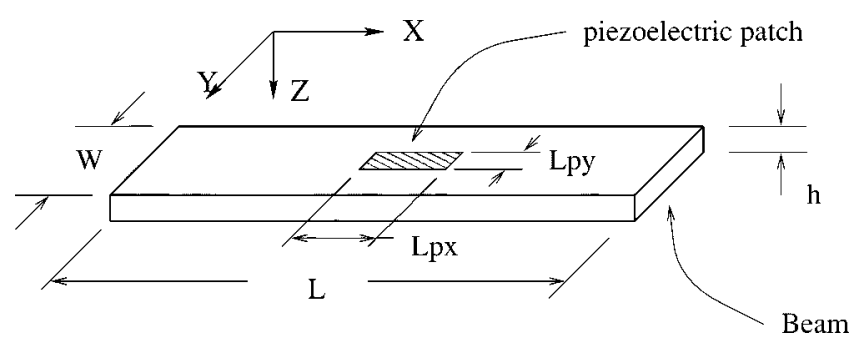

Fig. 2. A beam with a piezoelectric patch attached.

spatial, as well as, spectral properties of the system. The spatial information embedded in these models can then be used to design controllers which guarantee a certain level of damping for the entire structure.

Consider a homogeneous Euler-Bernoulli beam with dimensions of $L \times W \times h$ as in Fig. 2. The piezoelectric actuators and sensors have dimensions of $L_{p x} \times L_{p y} \times h_{p}$, where $h_{p}$ is the thickness of each patch. We denote the beam transverse deflection at point $x$ and at time $t$ by $z(x, t)$, assuming the beam as a one-dimensional system only. The PDE which governs the dynamics of the homogeneous beam is as follows [6], [14]:

$$
E I \frac{\partial^{4} z(x, t)}{\partial x^{4}}+\rho A_{b} \frac{\partial^{2} z(x, t)}{\partial t^{2}}=\frac{\partial^{2} M_{p x}(x, t)}{\partial x^{2}}
$$

where $\rho, A_{b}, E$, and $I$ represent density, cross-sectional area, Young's modulus of elasticity, and moment of inertia about the neutral axis of the beam, respectively. The moment acting on the beam is denoted by $M_{p x}$.

Initially, the relationship between the voltage applied to the piezoelectric patch and the moment generated by the patch needs to be obtained. The approach presented below follows the procedures in [7] and [8] and is included for the sake of completeness and clarity.

We consider the $i$ th piezoelectric actuator patch attached to the beam. The overall strain acting inside the actuator in the $X$ direction is a combination of the effect of the induced strain due to bending $\varepsilon_{x}$ and the unconstrained strain, $\varepsilon_{p}$. The unconstrained strain is the strain produced by the piezoceramic patch when the patch is free to expand or to contract [7], [8]. References [8] and [9] give an expression for the unconstrained strain (see Fig. 3) of the piezoelectric material due to the applied voltage

$$
\varepsilon_{p}=\left(\frac{d_{31}}{h_{p}}\right) V_{a i}(t)
$$




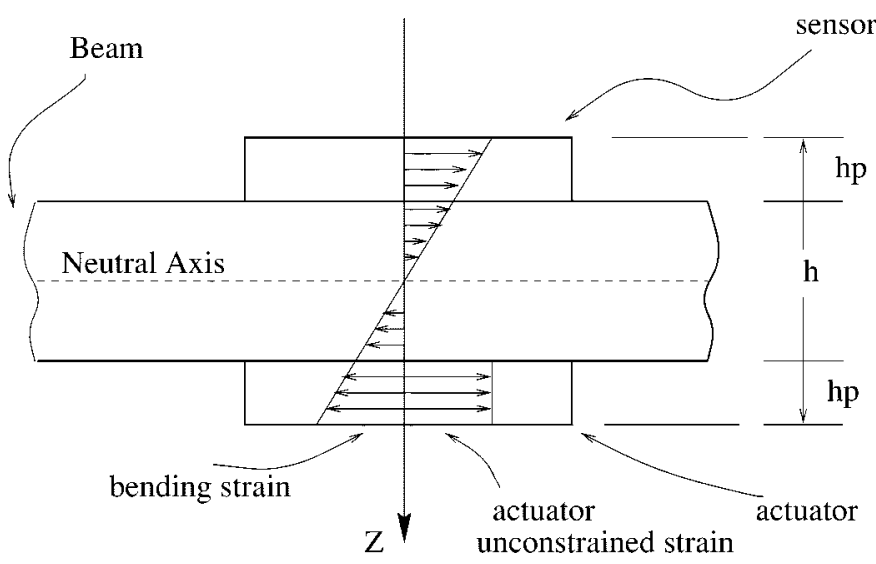

Fig. 3. Piezoelectric patches attached to a beam.

The piezoelectric charge constant is denoted by $d_{31}$, while the applied voltage to the $i$ th piezoelectric actuator is denoted by $V_{a i}(t)$.

We use Hooke's law to obtain an expression for stress in terms of strain

$$
\begin{aligned}
\sigma_{p x} & =E_{p}\left(\varepsilon_{x}-\varepsilon_{p}\right) \\
\sigma_{x} & =E \varepsilon_{x}
\end{aligned}
$$

where $\sigma_{p x}$ and $\sigma_{x}$ are the actuator axial stress and the beam axial stress in the $X$ direction, respectively, and $E_{p}$ is the Young's modulus of elasticity of the actuator.

The strain distribution across the beam thickness is assumed to be linear for pure bending case, $\varepsilon_{x}=\alpha z$, as shown in Fig. 3 . The strain gradient $\alpha$ is determined from the moment equilibrium equation about the beam's neutral axis

$$
\int_{-\frac{h}{2}}^{\frac{h}{2}} z \sigma_{x} d z+\int_{\frac{h}{2}}^{\frac{h}{2}+h_{p}} z \sigma_{p x} d z=0 .
$$

Here, we assume perfect bonding between the piezoelectric patch and the beam. This amounts to having no shearing effect in the patch-structure interface. We also assume that the neutral axis of the structure coincides with that of the beam alone and the sensor does not contribute to the structural properties. This is also a reasonable assumption if the patches are relatively much thinner than the beam's thickness.

From (5) and after some manipulation, the expression for the relationship between the unconstrained actuator strain $\varepsilon_{p}$ and the bending strain gradient $\alpha$ is found to be

$$
\alpha=\kappa \varepsilon_{p}
$$

where $\kappa=\left(12 E_{p} h_{p}\left(h_{p}+h\right)\right) /\left(2 E h^{3}+E_{p}\left[\left(h+2 h_{p}\right)^{3}-h^{3}\right]\right)$.

To incorporate the placement of the patch on the beam's surface in the $X$ direction, the use of step function $H(\cdot)$ is employed, where $H\left(x-x_{1 i}\right)$ is zero for $x<x_{1 i}$ and one for $x \geq x_{1 i}$. Here, $x_{1 i}$ and $x_{2 i}$ denote the location of the $i$ th piezoelectric patch's ends along the $X$ axis. Thus, the moment transferred to the beam from the piezoelectric actuator $M_{p x}$ can be obtained from the first integral term in (5) using (6)

$$
M_{p x}=\bar{K} V_{a i}(t)\left[H\left(x-x_{1 i}\right)-H\left(x-x_{2 i}\right)\right]
$$

where $\bar{K}=\left(\kappa E d_{31} h^{3} L_{p y} / 12 h_{p}\right)$ is a constant based on the properties of the beam and the piezoceramic patches. The forcing term in the PDE (1) can then be determined from the $M_{p x}$ expression, using the property of Dirac's delta function [15],

$$
\int_{-\infty}^{\infty} \delta^{(n)}(t-\theta) \phi(t) d t=(-1)^{n} \phi^{(n)}(\theta)
$$

where $\delta^{(n)}$ is the $n$th derivative of $\delta$ and $\phi$ is continuous at $\theta$.

Having obtained the piezoelectric moment expression, one needs to solve the PDE (1). The modal analysis technique is used for this purpose. We consider a solution of the form

$$
z(x, t)=\sum_{k=1}^{\infty} W_{k}(x) q_{k}(t)
$$

Here, $W_{k}(x)$ is determined from the eigenvalue problem, which is obtained by substituting (9) into (1) with no forcing function.

The eigenfunction $W_{k}(x)$ can be shown to have orthogonality properties

$$
\begin{aligned}
\int_{0}^{L} W_{k}(x) W_{p}(x) d x & =\delta_{k p} \\
\int_{0}^{L} \frac{E I}{\rho A_{b}} \frac{d^{4} W_{k}(x)}{d x^{4}} W_{p}(x) d x & =\omega_{k}^{2} \delta_{k p}
\end{aligned}
$$

where $\omega_{k}$ describes the natural frequency of the beam at mode $k$ and $\delta_{k p}$ is Kronecker's delta function.

A set of uncoupled ordinary differential equations (ODEs) can be obtained from the PDE (1) by using the orthogonality properties (10) and (11), and Dirac's delta function property (8) as well as the solution in (9). If the contribution of forcing functions generated by all $\bar{I}$ piezoelectric actuators is included, we obtain the following ODEs:

$$
\ddot{q}_{k}(t)+2 \zeta_{k} \omega_{k} \dot{q}_{k}(t)+\omega_{k}^{2} q_{k}(t)=\frac{\bar{K}}{\rho A_{b}} \sum_{i=1}^{\bar{I}} \Psi_{k i} V_{a i}(t)
$$

where $k=1,2, \ldots$ and $q_{k}(t)$ is the generalized coordinate of mode $k$. The subscript $i$ denotes the $i$ th actuator. It is assumed that all piezoelectric actuators and sensors that are used here are made from a similar material. A proportional damping term $2 \zeta_{k} \omega_{k}$ for each mode $k$ has been added into the equation of motion in (12). Further, $\Psi_{k i}$ can be obtained from

$$
\begin{aligned}
\Psi_{k i} & =\int_{0}^{L} W_{k}(x)\left[\frac{d \delta\left(x-x_{1 i}\right)}{d x}-\frac{d \delta\left(x-x_{2 i}\right)}{d x}\right] d x \\
& =\left[\frac{d W_{k}}{d x}\left(x_{2 i}\right)-\frac{d W_{k}}{d x}\left(x_{1 i}\right)\right] .
\end{aligned}
$$

Applying the Laplace transform to (12), assuming zero initial conditions, we obtain the multiple input-infinite output (MIIO) transfer function from the applied actuator-voltages, $V_{a}(s)=$ $\left[V_{a 1}(s), \ldots, V_{a \bar{I}}(s)\right]^{T}$, to the beam deflection $z(x, s)$ at location $x$

$$
G(s, x)=P \sum_{k=1}^{\infty} \frac{W_{k}(x) \bar{\Psi}_{k}^{T}}{s^{2}+2 \zeta_{k} \omega_{k} s+\omega_{k}^{2}}
$$


where $\bar{\Psi}_{k}=\left[\Psi_{k 1}, \ldots, \Psi_{k \bar{T}}\right]^{T}$. Here, $\Psi_{k i}$ is a function of the location of the $i$ th piezoelectric actuator-sensor pair and the eigenfunction, $W_{k}$. Also, $P$ is a constant that is dependent on the properties of the structure and the piezoceramic patches, i.e., $P=\left(\bar{K} / \rho A_{b}\right)$. We note that a similar methodology can be employed to obtain models of plates with certain boundary conditions.

\section{B. Piezoelectric Sensor}

In this paper, piezoelectric patches are used as sensors by placing the actuators and the sensors at the same location on both sides of the beam (i.e., we collocate the actuators and sensors). We consider the $l$ th piezoelectric sensor patch. Suppose the actuators produce forcing moments that vibrate the beam. This vibration induces strain inside the beam element. This strain will then induce an electric charge inside the piezoelectric sensor due to the piezoelectric effect. The electric charge distribution $q_{p}(t)$, i.e., the charge per unit length, for a dimensional structure is, as described in [10], given by

$$
q_{p}(t)=\frac{k_{31}^{2}}{g_{31}} \varepsilon_{x} L_{p y}
$$

where $k_{31}$ is the electromechanical coupling factor and $g_{31}$ is the piezoelectric voltage constant in the $X$ direction. Using Hooke's law for the beam deflection in the $X$ direction, $u$, the expression for the strain in the sensor patch is obtained as

$$
\varepsilon_{x}=\frac{\partial u}{\partial x}=-z_{p} \frac{\partial^{2} z}{\partial x^{2}} .
$$

where $z_{p}=-\left(h+h_{p} / 2\right)$ is the normal distance from the beam's neutral-axis to the midplane of the sensor patch. Here, we assume that the piezoelectric sensor is placed on the top surface of the beam as shown in Fig. 3.

The total electric charge can be obtained by integrating the expression $q_{p}(t)$ across the sensor's length (16). Substituting the solution form into (9), the induced sensor voltage $V_{s l}(t)$, at the $l$ th sensor, can be determined as

$$
V_{s l}(t)=\Upsilon \sum_{k=1}^{\infty} \int_{x_{1 l}}^{x_{2 l}} \frac{d^{2} W_{k}(x)}{d x^{2}} d x q_{k}(t)
$$

where $\Upsilon=\left(L_{p y} k_{31}^{2} / C g_{31}\right)\left(h+h_{p} / 2\right)$ and $C$ is the capacitance of the piezoelectric sensor. The integral given in the above equation is equal to $\Psi_{k i}$ in (14) when $i$ is replaced by $l$. This equation implies that the sensor output consists of the contribution of each mode, where each mode contribution is proportional to $\Psi_{k l}$.

Taking the Laplace transform of (12) and (18), the multiple input-multiple output (MIMO) transfer function from the applied actuator-voltages, $V_{a}(s)$, to the induced voltages at the sensors, $V_{s}(s)=\left[V_{s 1}(s), \ldots, V_{s \bar{I}}(s)\right]^{T}$, can be written as

$$
G_{V s}(s)=P_{V s} \sum_{k=1}^{\infty} \frac{\bar{\Psi}_{k} \bar{\Psi}_{k}^{T}}{s^{2}+2 \zeta_{k} \omega_{k} s+\omega_{k}^{2}}
$$

where $P_{V s}>0$ is a constant determined by the properties of the structure and of the piezoceramic patches, i.e., $P_{V s}=\Upsilon P$. To this end, we note that for more complicated structures, such as plates with certain boundary conditions, similar transfer functions can be obtained.

\section{SPATIAL $\mathcal{H}_{2}$ NORM}

This section presents an overview of the concept of spatial $\mathcal{H}_{2}$ norm that will be used in the optimization procedure in Section V. Consider the transfer function of a flexible structure, $G(s, x)$, as in (15). This model describes the structural deflection at some point $x$. The $\mathcal{H}_{2}$ norm of $G(s, x)$ can be used as a measure of performance at the point $x$. However, the $\mathcal{H}_{2}$ norm of $G(s, x)$ cannot be used as a global performance measure since it is calculated based on the response at a specific location on the structure [5], [13]. To overcome this difficulty, the notion of spatial $\mathcal{H}_{2}$ norm was introduced in [5] and [13].

The spatial $\mathcal{H}_{2}$ norm of the transfer function $G(s, x)$ is defined [5], [13] as

$$
\langle\langle G\rangle\rangle_{2}^{2}=\frac{1}{2 \pi} \int_{-\infty}^{\infty} \int_{X} \operatorname{tr}\left\{G(j \omega, x)^{*} G(j \omega, x)\right\} d x d \omega
$$

where $X$ is the set over which $x$ is defined. For a beam, $X=$ $[0, L]$. Here, $\operatorname{tr}\{F\}$ represents the trace of the matrix $F$. Taking advantage of the orthonormality of the eigenfunctions, $W_{k}$, in (10), it can be shown that

$$
\left\langle\langle G\rangle_{2}^{2}=\sum_{k=1}^{\infty}\left\|\tilde{G}_{k}\right\|_{2}^{2}\right.
$$

where

$$
\tilde{G}_{k}(s)=P \frac{\bar{\Psi}_{k}^{T}}{s^{2}+2 \zeta_{k} \omega_{k}+\omega_{k}^{2}} .
$$

From definition (20), it can be argued that the spatial $\mathcal{H}_{2}$ norm is a suitable measure of performance for spatially distributed linear time invariant systems, such as those described in (15). Furthermore, if the system can be broken into a number of orthonormal modes, then the contribution of each mode to the total spatial $\mathcal{H}_{2}$ norm of the system can be determined from (21) and (22). Also, since the spatial $\mathcal{H}_{2}$ norm of a system of the form (15) is equivalent to the $\mathcal{H}_{2}$ norm of a finite-dimensional system, it can be calculated using standard software.

Another way of calculating the spatial $\mathcal{H}_{2}$ norm of a system is to first obtain a state-space representation of the system and then to apply definition (20) to determine a finite-dimensional system whose $\mathcal{H}_{2}$ norm is equivalent to the spatial $\mathcal{H}_{2}$ norm of the spatially distributed system. To demonstrate this, a state-space representation of the transfer function $G(s, x)$ can be described as

$$
\begin{aligned}
\dot{x}_{b}(t) & =A_{d} x_{b}(t)+B_{d} \bar{w}(t) \\
z(x, t) & =C_{d}(x) x_{b}(t)
\end{aligned}
$$

where $\bar{w}(t)$ are external disturbances through the actuators. Notice that for a vibratory system such as a beam, $z(x, t)$ represents the deflection at a particular point, $x$, along the structure. Such a model can be obtained by truncating the series (15) and keeping the first $N$ modes. Then the spatial $\mathcal{H}_{2}$ norm of the transfer function $G(s, x)$ can be shown to be equivalent to ([5], [13])

$$
\langle\langle G\rangle\rangle_{2}^{2}=\|\hat{G}\|_{2}^{2}
$$


where

$$
\hat{G}(s)=\Gamma\left(s I-A_{d}\right)^{-1} B_{d}
$$

and,

$$
\Gamma^{T} \Gamma=\int_{0}^{L} C_{d}(x)^{T} C_{d}(x) d x
$$

Hence, the spatial $\mathcal{H}_{2}$ norm of $G(s, x)$ can be determined by calculating the $\mathcal{H}_{2}$ norm of $\hat{G}(s)$.

\section{Controller Structure}

In this section, we present a multivariable controller structure that is applicable to flexible structures consisting of highly resonant modes with compatible and collocated actuators and sensors. Moreover, we will establish closed-loop stability in the presence of uncontrolled in-bandwidth and out-of-bandwidth modes. In the sequel, however, we concentrate on piezoelectric laminate structures.

Let us consider the feedback control loop shown in Fig. 1. The system is represented as a flexible structure with $\bar{I}$ collocated piezoelectric actuator-sensor pairs attached to it. The $i$ th actuator-sensor pair is controlled independently by the controller $K_{i}$. The measured voltages from the piezoelectric sensors, $V_{s}(t)$, act as the measured inputs to the controller $K(s)$, while the controller applies voltages to the piezoelectric actuators as, $m(t)=\left[m_{1}, \ldots, m_{\bar{I}}\right]^{T}$. We use $\bar{w}(t)=\left[\bar{w}_{1}, \ldots, \bar{w}_{\bar{I}}\right]^{T}$ to signify external disturbances acting on the structure, through the actuators.

A significant response of a flexible structure occurs only when the excitation frequency is near a natural frequency of the system. At these, so called resonant frequencies, the responses are rather significant. This is mainly due to the fact that these structures are often very lightly damped. Hence, the task of minimizing the structural vibration can be considered to be that of minimizing the resonant responses which occur at, or very close to, the structure's natural frequencies. An ideal controller would significantly reduce the structural vibration at, and near to, the resonant frequencies of the structure. However, it should have limited effect at other frequencies.

A class of controllers that can achieve good damping levels and avoid instability in the face of spill-over dynamics, is presented here. These controllers apply high gain feedback at resonant frequencies but have limited effect at other frequencies. The controllers have a decentralized nature, and are defined as

$$
K(s)=\operatorname{diag}\left(K_{1}(s), K_{2}(s), \ldots, K_{\bar{I}}(s)\right)
$$

where

$$
\begin{array}{r}
K_{i}(s)=\sum_{k=1}^{N}\left(\frac{\alpha_{i k} s\left(s+2 d_{i k} \omega_{k}\right)}{s^{2}+2 d_{i k} \omega_{k} s+\omega_{k}^{2}}+R_{i k}\right) \\
i=1,2, \ldots, \bar{I}, \quad \alpha_{i k} \geq 0 \quad \forall i, k .
\end{array}
$$

Here, we assume that $N$ modes lie within the controlled bandwidth and out of these modes, $N_{c} \leq N$ modes are to be controlled. The term $\alpha_{i k}$ signifies the $k$ th modal gain of the controller for the $i$ th actuator-sensor pair and $R_{i k}$ is an arbitrary constant. If a particular mode is not to be controlled, then $\alpha_{i k}$

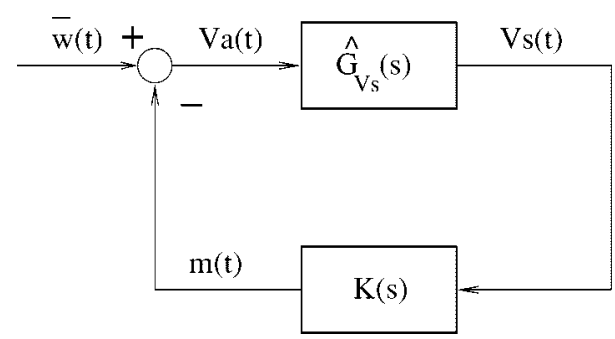

(a)

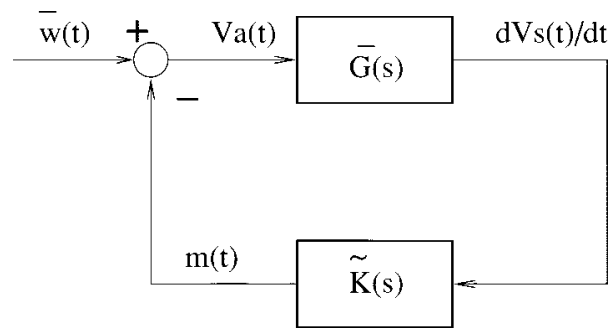

(b)

Fig. 4. Feedback systems. (a) Negative feedback loop. (b) Equivalent feedback system.

and $R_{i k}$ corresponding to that mode are simply set to zero for all $i$. Otherwise, if a mode is to be controlled, $\alpha_{i k}$ corresponding to that mode is assigned a positive number, i.e., $\alpha_{i k}>0$ for all $i$.

The resonant controller structure proposed in [1] can be shown to be a special case of our resonant controller structure when $R_{i k}=0$ for all $k$ and $i=1$, i.e., an SISO controller. The particular type of controller structure described here increases the damping of the system at the resonant frequencies, which will result in the reduction of the resonant responses.

To this end, it is clear that this particular controller structure should have a highly resonant nature, so that it applies a high gain at each resonant frequency of the structure that is to be controlled. Hence, if control parameters $\alpha_{i k}, d_{i k}$, and $R_{i k}$ are chosen appropriately, the closed-loop frequency response of the system can be considerably damped at, and close to, the resonant frequencies. Furthermore, this controller structure is appealing since it has minimal effect on high-frequency spill-over dynamics. We will prove that this particular class of controllers is stabilizing and maintains stability in the presence of high-frequency uncontrolled dynamics and imperfect models.

Consider the negative feedback connection shown in Fig. 4(a). Here, we assume that $K(s)$ has a structure defined by (27) and (28). We also assume that $\hat{G}_{V s}(s)$ is a truncated version of (19). That is,

$$
\hat{G}_{V s}(s)=P_{V s} \sum_{k=1}^{M} \frac{\bar{\Psi}_{k} \bar{\Psi}_{k}^{T}}{s^{2}+2 \zeta_{k} \omega_{k} s+\omega_{k}^{2}}
$$

where $M \gg N$ and $\omega_{N}$ is the highest resonant mode that is to be controlled. We should point out that we need to work with a finite-dimensional model of $\hat{G}_{V s}(s)$ since we intend to use stability results that are applicable to finite-dimensional linear time-invariant systems. We also point out that it is acceptable to work with a truncated version of (19) since the transfer function (19) rolls off at higher frequencies [16]. Therefore, for large enough $M$, the stability results will extend to the full infinitedimensional model of the system. 
To prove closed-loop stability of the negative feedback loop in Fig. 4(a), we consider the equivalent feedback system shown in Fig. 4(b) where

$$
\bar{G}(s)=s \hat{G}_{V s}(s)
$$

and

$$
\tilde{K}(s)=\frac{K(s)}{s} .
$$

Notice that $\bar{G}(s)$ is the transfer function from the actuator voltage to the rate of change of the sensor voltage $d V_{s}(t) / d t$.

We establish that $\bar{G}(s)$ is a positive-real transfer function, by showing that $\bar{G}(j \omega)+\bar{G}(j \omega)^{*}$ is positive-semidefinite for all $\omega$. That is,

$$
\begin{aligned}
\bar{G}(j \omega)+\bar{G}(j \omega)^{*}= & P_{V s} \sum_{k=1}^{M}\left(\frac{j \bar{\Psi}_{k} \bar{\Psi}_{k}^{T} \omega}{\omega_{k}^{2}+j 2 \zeta_{k} \omega_{k} \omega-\omega^{2}}\right. \\
& \left.+\frac{-j \bar{\Psi}_{k} \bar{\Psi}_{k}^{T} \omega}{\omega_{k}^{2}-j 2 \zeta_{k} \omega_{k} \omega-\omega^{2}}\right) \\
= & P_{V s} \sum_{k=1}^{M} \frac{4 \bar{\Psi}_{k} \bar{\Psi}_{k}^{T} \zeta_{k} \omega_{k} \omega^{2}}{\left(\omega_{k}^{2}-\omega^{2}\right)^{2}+\left(2 \zeta_{k} \omega_{k} \omega\right)^{2}} \\
\geq & 0 \quad \omega \in \mathbf{R} .
\end{aligned}
$$

Having established the positive-realness of $\bar{G}(s)$, closed-loop stability follows if we can show that $\tilde{K}(s)$ is strictly positive real in the weak sense, see, e.g., [17] (A stable square transfer function matrix $\tilde{K}(s)$ is strictly positive-real in the weak sense if $\tilde{K}(j \omega)+\tilde{K}(j \omega)^{*}>0$ for $\left.\omega \in(-\infty, \infty)\right)$.

Noticing that $\tilde{K}(s)$ is diagonal, we need only consider its diagonal elements. We have

$$
\begin{aligned}
& \tilde{K}_{i}(j \omega)+\tilde{K}_{i}(j \omega)^{*} \\
& =\sum_{k=1}^{N}\left\{\alpha _ { i k } \left(\frac{j \omega+2 d_{i k} \omega_{k}}{\omega_{k}^{2}+j 2 d_{i k} \omega_{k} \omega-\omega^{2}}\right.\right. \\
& \left.\left.\quad+\frac{-j \omega+2 d_{i k} \omega_{k}}{\omega_{k}^{2}-j 2 d_{i k} \omega_{k} \omega-\omega^{2}}\right)+\left(\frac{R_{i k}}{j \omega}+\frac{R_{i k}}{-j \omega}\right)\right\} \\
& =\sum_{k=1}^{N} \frac{4 \alpha_{i k} d_{i k} \omega_{k}^{3}}{\left(\omega_{k}^{2}-\omega^{2}\right)^{2}+\left(2 d_{i k} \omega_{k} \omega\right)^{2}} \\
& >0 \quad \omega \in(-\infty, \infty), \quad i=1,2, \ldots, \bar{I} .
\end{aligned}
$$

This implies that $\tilde{K}(j \omega)=\operatorname{diag}\left(\tilde{K}_{1}(j \omega), \ldots, \tilde{K}_{\bar{I}}(j \omega)\right)>0$ for $\omega \in(-\infty, \infty)$. Therefore, using Corollary 1.1 of [17], the negative feedback connection of $\tilde{K}(s)$ and $\bar{G}(s)$ is stable. Hence, the negative feedback connection of Fig. 4 is stable.

Note that the class of controllers defined by (27) and (28) is robustly stable with respect to unknown resonant frequencies $\omega_{k}$ and damping factors $\zeta_{k}$. Indeed, as long as the actuators and sensors are collocated, positive-realness of $\bar{G}(s)$ is guaranteed. Hence, any controller $K(s)$ with $\tilde{K}(s)=(K(s) / s)$ such that $\tilde{K}(s)$ is strictly positive-real in the weak sense will ensure closed-loop stability. Furthermore, stability is guaranteed in the presence of high-frequency and in-bandwidth modes which are left uncontrolled. Moreover, if the resonant frequencies and damping ratios of the model are incorrect, the controller will not destabilize the closed-loop system. However, under these conditions, it will most likely have poor performance.

Having established stability properties of the resonant controller, we now proceed to determining controller parameters via an optimization procedure.

\section{OPTIMIZATION FOR RESONANT CONTROLLERS}

One of the interesting properties of the class of controllers proposed here is that they are highly resonant. Given that the structure is also highly resonant and the peaks are reasonably far away from one another, this would mean that the controller effect is localized to the resonant peaks. Moreover, in order to control each mode, one would only need to determine three parameters, i.e., $\alpha_{i k}, d_{i k}$, and $R_{i k}$. For an SISO system this could be easily done by trial and error. However, for a multivariable plant, a more systematic method is desirable. In this section, we show how these parameters can be determined by solving an optimization problem.

From (28) it can be observed that the controller $K(s)$ is parameterized in terms of $\alpha_{i k}, d_{i k}$, and $R_{i k}$. Therefore, any optimization has to be carried out over these parameters. Our approach, however, is to choose a set of parameters $\alpha_{i k}$ and $R_{i k}$ first and then optimize over $d_{i k}$.

The state-space form of a flexible structure based on the truncated version of $G(s, x)$ in (15) and $G_{V s}(s)$ in (19) can be written in the form of

$$
\begin{aligned}
\dot{x}_{b}(t) & =\left[\begin{array}{cc}
0 & I \\
-W_{b}^{2} & -2 Z_{b} W_{b}
\end{array}\right] x_{b}(t)+\left[\begin{array}{c}
0 \\
H
\end{array}\right] \bar{w}(t) \\
z(x, t) & =\left[\begin{array}{ll}
U_{d}(x) & 0
\end{array}\right] x_{b}(t) \\
V_{s}(t) & =\left[\begin{array}{ll}
U & 0
\end{array}\right] x_{b}(t)
\end{aligned}
$$

where

$$
\begin{aligned}
Z_{b} & =\operatorname{diag}\left(\zeta_{1}, \ldots, \zeta_{M}\right) \\
W_{b} & =\operatorname{diag}\left(\omega_{1}, \ldots, \omega_{M}\right) \\
H & =P\left[\bar{\Psi}_{1}, \ldots, \bar{\Psi}_{M}\right]^{T} \\
U & =\Upsilon\left[\bar{\Psi}_{1}, \ldots, \bar{\Psi}_{M}\right] \\
U_{d}(x) & =\left[W_{1}(x), \ldots, W_{M}(x)\right] .
\end{aligned}
$$

The number of modes considered in the truncated model is represented by $M$. The state $x_{b}$ is $\left[q_{1}, \ldots, q_{M}, \dot{q}_{1}, \ldots, \dot{q}_{M}\right]^{T}$. Again, $V_{s}=\left[V_{s 1}, \ldots, V_{s \bar{I}}\right]^{T}$ are the voltage measurements from the piezoelectric sensors and $z(x, t)$ is the transverse deflection at a particular point $x$ along the beam.

For a MIMO system, each independent controller $K_{i}(s)$ given in (28) can be written in its state-space form

$$
\begin{aligned}
\dot{\hat{x}}_{i}(t) & =\left[\begin{array}{cc}
0 & I \\
-W_{c}^{2} & -2 D_{i} W_{c}
\end{array}\right] \hat{x}_{i}(t)+\left[\begin{array}{c}
0 \\
U_{i}^{\prime}
\end{array}\right] V_{s i}(t) \\
m_{i}(t) & =\left[\begin{array}{ll}
\bar{U}_{i} & 0
\end{array}\right] \hat{x}_{i}(t)+V_{i} V_{s i}(t) .
\end{aligned}
$$

If we order the vibration modes that are to be controlled as $n_{1}, \ldots, n_{N_{c}}$, where $N_{c}$ is the number of modes to be controlled, we then define: $D_{i}=$ diag $\left(d_{i n_{1}}, \ldots, d_{i n_{N_{c}}}\right), W_{c}=\operatorname{diag}\left(\omega_{n_{1}}, \ldots, \omega_{n_{N_{c}}}\right)$ and $\hat{x}_{i}=\left[\hat{q}_{i n_{1}}, \ldots, \hat{q}_{i n_{N_{c}}}, \dot{\hat{q}}_{i n_{1}}, \ldots, \dot{\hat{q}}_{i n_{N_{c}}}\right]^{T}$. The terms $U_{i}^{\prime}, \bar{U}_{i}$, and $V_{i}$ can also be obtained in a straightforward manner. 
Hence, the MIMO controller can be described as follows:

$$
\begin{aligned}
\dot{\hat{x}}(t) & =\left[\begin{array}{cc}
0 & I \\
-\tilde{W}^{2} & -2 \tilde{D} \tilde{W}
\end{array}\right] \hat{x}(t)+\left[\begin{array}{c}
0 \\
\tilde{U}^{\prime}
\end{array}\right] V_{s}(t) \\
m(t) & =\left[\begin{array}{ll}
\tilde{\bar{U}} & 0
\end{array}\right] \hat{x}(t)+\tilde{V} V_{s}(t)
\end{aligned}
$$

where

$$
\begin{aligned}
\tilde{W} & =\operatorname{diag}\left(W_{c}, \ldots, W_{c}\right) \\
\tilde{D} & =\operatorname{diag}\left(D_{1}, \ldots, D_{\bar{I}}\right) \\
\tilde{U}^{\prime} & =\operatorname{diag}\left(U_{1}^{\prime}, \ldots, U_{\bar{I}}^{\prime}\right) \\
\tilde{\bar{U}} & =\operatorname{diag}\left(\bar{U}_{1}, \ldots, \bar{U}_{\bar{I}}\right) \\
\tilde{V} & =\operatorname{diag}\left(V_{1}, \ldots, V_{\bar{I}}\right)
\end{aligned}
$$

and the controller state

$$
\begin{aligned}
\hat{x}= & {\left[\hat{q}_{1 n_{1}}, \hat{q}_{1 n_{2}}, \ldots, \hat{q}_{\bar{I}}{ }_{n_{\left(N_{c}-1\right)}}, \hat{q}_{\bar{I}_{n_{N_{c}}}}, \dot{\hat{q}}_{1 n_{1}}, \dot{\hat{q}}_{1 n_{2}}, \ldots\right.} \\
& \left.\dot{\hat{q}}_{\bar{I}_{n_{\left(N_{c}-1\right)}}, \ldots}, \hat{\hat{q}}_{\bar{I} n_{N_{c}}}\right]^{T} .
\end{aligned}
$$

Here, $m=\left[m_{1}, \ldots, m_{\bar{I}}\right]^{T}$ are the output voltages from the controller.

The closed-loop system can then be obtained from (34) and (37) as follows:

$$
\begin{aligned}
\dot{\bar{x}}(t) & =\bar{A} \bar{x}(t)+\bar{B} \bar{w}(t) \\
z(x, t) & =\bar{C}_{d}(x) \bar{x}(t) \\
V_{s}(t) & =\bar{C} \bar{x}(t)
\end{aligned}
$$

where $\bar{x}=\left[\begin{array}{ll}x_{b}^{T} & \hat{x}^{T}\end{array}\right]^{T}$ and

$$
\begin{aligned}
\bar{A} & =\left[\begin{array}{cccc}
0 & I & 0 & 0 \\
-\tilde{W}^{2}-H \tilde{V} U & -2 Z_{b} W_{b} & -H \bar{U} & 0 \\
0 & 0 & 0 & I \\
\tilde{U}^{\prime} U & 0 & -\tilde{W}^{2} & -2 \tilde{D} \tilde{W}
\end{array}\right] \\
\bar{B} & =\left[\begin{array}{llll}
0 & H & 0 & 0
\end{array}\right]^{T} \\
\bar{C} & =\left[\begin{array}{llll}
U & 0 & 0 & 0
\end{array}\right] \\
\bar{C}_{d}(x) & =\left[\begin{array}{llll}
U_{d}(x) & 0 & 0 & 0
\end{array}\right]
\end{aligned}
$$

where 0 and $I$ are the zero and identity matrices with the appropriate sizes, respectively.

The matrix $\bar{A}$ can be represented as a finite sum of the following form:

$$
\bar{A}=A-2 \sum_{i=1}^{\bar{I}} \sum_{j=1}^{N_{c}} \omega_{n_{j}} d_{i n_{j}} E_{i n_{j}} E_{i n_{j}}^{T}
$$

where $A$ is independent of damping ratio $d_{i k}$.

Here, the use of $k$ and $n_{j}$ should not be confused since $n_{j}$ is used to specify each vibration mode that is to be controlled in the optimization process. This notation is adopted here since only a limited number of in-bandwidth modes are to be controlled. However, we will use $k$ to signify vibration modes in the general case throughout this paper.
The term $E_{i n_{j}}$ is defined as

$$
E_{i n_{j}}=\left[\begin{array}{c}
0_{M \times 1} \\
0_{M \times 1} \\
0_{\left(\bar{I} N_{c}\right) \times 1} \\
\bar{E}_{i n_{j}\left(\bar{I} N_{c}\right) \times 1}
\end{array}\right] .
$$

Here, $\bar{E}_{i n_{j}}$ consists of zero elements except for the corresponding row of $d_{i n_{j}}$, i.e. $\bar{E}_{i n_{j}}\left((i-1) N_{c}+j, 1\right)=1$. Notice that for fixed values of $\alpha_{i k}$ and $R_{i k}$, the system (40) is parameterized in terms of $d_{i k}$.

In order to obtain a suitable set of $d_{i k}$ parameters, we propose the following optimization problem:

$$
d_{i k}^{*}=\arg \min \left\langle\left\langle T_{z \bar{w}}(s, x)\right\rangle_{2}^{2}\right.
$$

where $T_{z \bar{w}}(s, x)$ represents the closed-loop transfer function from $\bar{w}$ to $z$. This describes the minimization of the spatial $\mathcal{H}_{2}$ norm of the closed-loop transfer function from input disturbances $\bar{w}$ to every point on the structure $z$. Hence, we can obtain a controller that can minimize structural vibration in a spatially averaged sense.

In Section III we showed that the spatial $\mathcal{H}_{2}$ norm of (40) is equivalent to the $\mathcal{H}_{2}$ norm of the following finite-dimensional system:

$$
\begin{aligned}
\dot{\bar{x}}(t) & =\bar{A} \bar{x}(t)+\bar{B} \bar{w}(t) \\
\bar{z}(t) & =\bar{\Gamma} \bar{x}(t) \\
V_{s}(t) & =\bar{C} \bar{x}(t)
\end{aligned}
$$

where $\bar{\Gamma}$ is calculated from

$$
\bar{\Gamma}^{T} \bar{\Gamma}=\int_{0}^{L} \bar{C}_{d}(x)^{T} \bar{C}_{d}(x) d x .
$$

The optimization problem (44) can then be rewritten as

$$
\begin{array}{ll}
\min & \operatorname{tr}\left(\bar{B}^{T} L_{o} \bar{B}\right) \\
\text { subject to: } & \bar{A}^{T} L_{o}+L_{o} \bar{A}+\bar{\Gamma}^{T} \bar{\Gamma}=0 .
\end{array}
$$

Notice that here $\bar{A}$ depends on $d_{i k}$ via (42).

We point out that for a fixed value for $\alpha_{i k} \mathrm{~s}$, it is not possible to make the cost function arbitrarily small. Indeed, if $d_{i k} \rightarrow \infty$, it can be shown that the controller in (28) reduces to a simple gain. In that case, the only way that the cost function can be made arbitrarily small, is by making $\alpha_{i k}$ 's arbitrarily large. This is not an appropriate solution for the reasons that will be explained in the sequel.

To find a solution to this constrained optimization problem, we introduce a matrix of Lagrange multipliers $S$ and form the Lagrangian as follows, incorporating (42)

$$
\begin{aligned}
L= & \operatorname{tr}\left(\bar{B}^{T} L_{o} \bar{B}\right)+\operatorname{tr}\left\{\left(\bar{A}^{T} L_{o}+L_{o} \bar{A}+\bar{\Gamma}^{T} \bar{\Gamma}\right) S\right\} \\
= & \operatorname{tr}\left(\bar{B}^{T} L_{o} \bar{B}\right)+\operatorname{tr}\left\{A^{T} L_{o} S+L_{o} A S+\bar{\Gamma}^{T} \bar{\Gamma} S\right. \\
& -2 \sum_{i=1}^{\bar{I}} \sum_{j=1}^{N_{c}} \omega_{n_{j}} d_{i n_{j}} E_{i n_{j}} E_{i n_{j}}^{T} L_{o} S \\
& \left.-2 L_{o} \sum_{i=1}^{\bar{I}} \sum_{j=1}^{N_{c}} \omega_{n_{j}} d_{i n_{j}} E_{i n_{j}} E_{i n_{j}}^{T} S\right\} .
\end{aligned}
$$


First-order necessary conditions for optimality can be obtained by taking the derivatives of the Lagrangian $L$, with respect to parameters $L_{o}, S$, and $d_{i n_{j}}$ and setting the derivatives to zero. That is

$$
\begin{aligned}
\frac{\partial L}{\partial L_{o}} & =\bar{B} \bar{B}^{T}+\bar{A} S+S \bar{A}^{T}=0 \\
\frac{\partial L}{\partial S} & =\bar{A}^{T} L_{o}+L_{o} \bar{A}+\bar{\Gamma}^{T} \bar{\Gamma}=0 \\
\frac{\partial L}{\partial d_{i n_{j}}} & =-4 \omega_{n_{j}} E_{i n_{j}}^{T} L_{o} S E_{i n_{j}}=0, \\
& j=1,2, \ldots, N_{c} \quad i=1,2, \ldots, \bar{I} .
\end{aligned}
$$

To obtain the minimum of the $\mathcal{H}_{2}$ norm of the system, (49)-(51) have to be solved simultaneously. However, it is not possible to obtain such a closed-form solution, so a numerical approach has to be used instead. The form given in (51) can be used as the gradient to obtain a local minimum of the function.

To start the optimization procedure an initial guess for each damping ratio, $d_{i k}$ (or its corresponding $d_{i n_{j}}$ ), has to be made. Any positive $d_{i k}$ can be used as a starting point since that would guarantee stability. Here, the modal gains, $\alpha_{i k}$, and $R_{i k}$ are set at some specific levels to obtain a sufficient reduction of each resonant response. It is, however, possible to optimize over $\alpha_{i k}$ and $R_{i k}$ as well as $d_{i k}$.

Matrices $\bar{B}$ and $\bar{\Gamma}$ only need to be calculated once, because they are independent of damping variables $d_{i k}$. Matrix $\bar{A}$ is obtained from (42), while the observability and controllability Grammian matrices, $L_{\circ}$ and $S$, are calculated by solving the Lyapunov equations in (49) and (50). The gradient for each value of damping $d_{i k}$ is calculated from (51). The process is iterated by updating the damping ratio $d_{i k}$ until a solution with acceptable accuracy is obtained. Furthermore, since the optimization problem is nonconvex in general, the iterative optimization procedure can be carried out for a number of initial guesses, and then the best solution can be used.

To make the optimization concept clearer, we will demonstrate the use of this optimization process for our resonant controller in the next section.

\section{Spatial Resonant Control of a PiEzoElectric LAMINATE BEAM}

In this section, we show how the proposed resonant controller can be implemented to dampen vibration of a piezoelectric laminate beam.

A simply supported flexible beam-such as the one shown in Fig. 1-with a collocated piezoelectric actuator-sensor pair attached to it is used in the experiments. The structure consists of a $60 \mathrm{~cm}$ long uniform aluminum bar of rectangular cross section $(50 \mathrm{~mm} \times 3 \mathrm{~mm})$. The beam is pinned at both ends. A pair of piezoelectric ceramic elements are attached symmetrically to either side of the beam, $50 \mathrm{~mm}$ away from one end of the beam. The piezoceramic elements used in our experiment are PIC151 patches. These patches are $25 \mathrm{~mm}$ wide, $70 \mathrm{~mm}$ long, and $0.25 \mathrm{~mm}$ thick. The physical parameters of PIC151 are given in Table I.

A model of the composite structure is obtained via modal analysis as explained in Section II. The model is truncated by
TABLE I

Properties OF THE PIEZOELECTRIC LAMINATE BEAM

\begin{tabular}{l|l}
\hline \hline Beam $X$-length, $L$ & $0.600 \mathrm{~m}$ \\
\hline Beam width, $W$ & $0.050 \mathrm{~m}$ \\
\hline Beam thickness, $h$ & $0.003 \mathrm{~m}$ \\
\hline Beam Young's Modulus, $E$ & $7.00 \times 10^{10} \mathrm{~N} / \mathrm{m}^{2}$ \\
\hline Beam density, $\rho$ & $2.770 \times 10^{3} \mathrm{~kg} / \mathrm{m}^{3}$ \\
\hline \hline Piezoceramic $X$-length, $L_{p x}$ & $0.070 \mathrm{~m}$ \\
\hline Piezoceramic $Y$-length, $L_{p y}$ & $0.025 \mathrm{~m}$ \\
\hline Piezoceramic thickness, $h_{p}$ & $2.50 \times 10^{-4} \mathrm{~m}$ \\
\hline Piezoceramic Young's Modulus, $E_{p}$ & $6.70 \times 10^{10} \mathrm{~N} / \mathrm{m}^{2}$ \\
\hline Charge constant, $d_{31}$ & $-2.10 \times 10^{-10} \mathrm{~m} / \mathrm{V}$ \\
\hline Voltage constant, $g_{31}$ & $-1.15 \times 10^{-2} \mathrm{Vm} / \mathrm{N}$ \\
\hline Capacitance, $C$ & $1.05 \times 10^{-7} \mathrm{~F}$ \\
\hline Electromechanical coupling factor, $k_{31}$ & 0.34 \\
\hline \hline
\end{tabular}

keeping the first ten structural modes, i.e., $M=10$. However, the effect of out-of-bandwidth modes has to be taken into consideration to correct the location of the in-bandwidth zeros of the truncated model as discussed in [18]-[20].

To reduce the errors of the in-bandwidth zeros, we add feedthrough terms, $D_{d}(x)$ and $D_{V s}$, to the system outputs in (34), $z(x, t)$ and $V_{s}(t)$, as follows:

$$
\begin{aligned}
\dot{x}_{b}(t) & =A_{d} x_{b}(t)+B_{d} \bar{w}(t) \\
z(x, t) & =C_{d}(x) x_{b}(t)+D_{d}(x) \bar{w}(t) \\
V_{s}(t) & =C_{V s} x_{b}(t)+D_{V s} \bar{w}(t)
\end{aligned}
$$

where $A_{d}, B_{d}, C_{d}$, and $C_{V s}$ are defined in (34). We can rely on experiments to estimate these feedthrough terms. However, estimation of feedthrough term $D_{d}(x)$ can be impractical because the term is a function of spatial location $x$. Further, the spatial integration that is needed to calculate the system's spatial $\mathcal{H}_{2}$ norm (26) will be tedious. Thus, we decide to use the method proposed in [19] to estimate the value of $D_{d}(x)$. Also, the feedthrough term $D_{V s}$ can be determined via a procedure similar to the one described in [21].

The feedthrough term $D_{d}(x)$ is calculated to minimize the spatial $\mathcal{H}_{2}$ norm of the error between the infinite-dimensional model and the truncated model [19].

$$
D_{d}(x)=\sum_{k=M+1}^{M_{\max }} K_{k}^{\mathrm{opt}} W_{k}(x)
$$

where $K_{k}^{\text {opt }}$ can be calculated as in [19]

$$
K_{k}^{\mathrm{opt}}=\frac{1}{2 \omega_{c o} \omega_{k}} \ln \left(\frac{\omega_{k}+\omega_{c o}}{\omega_{k}-\omega_{c o}}\right) P \Psi_{k 1} .
$$

Here, $\omega_{c o}$ is chosen to lie within the interval $\omega_{c o} \in\left(\omega_{M}, \omega_{M+1}\right)$ and $P$ and $\Psi_{k 1}$ are defined in (15) and (14), respectively. The above term is calculated by considering modes $M+1$ to $M_{\max }=200$ to obtain a reasonable approximation to the feedthrough term. 


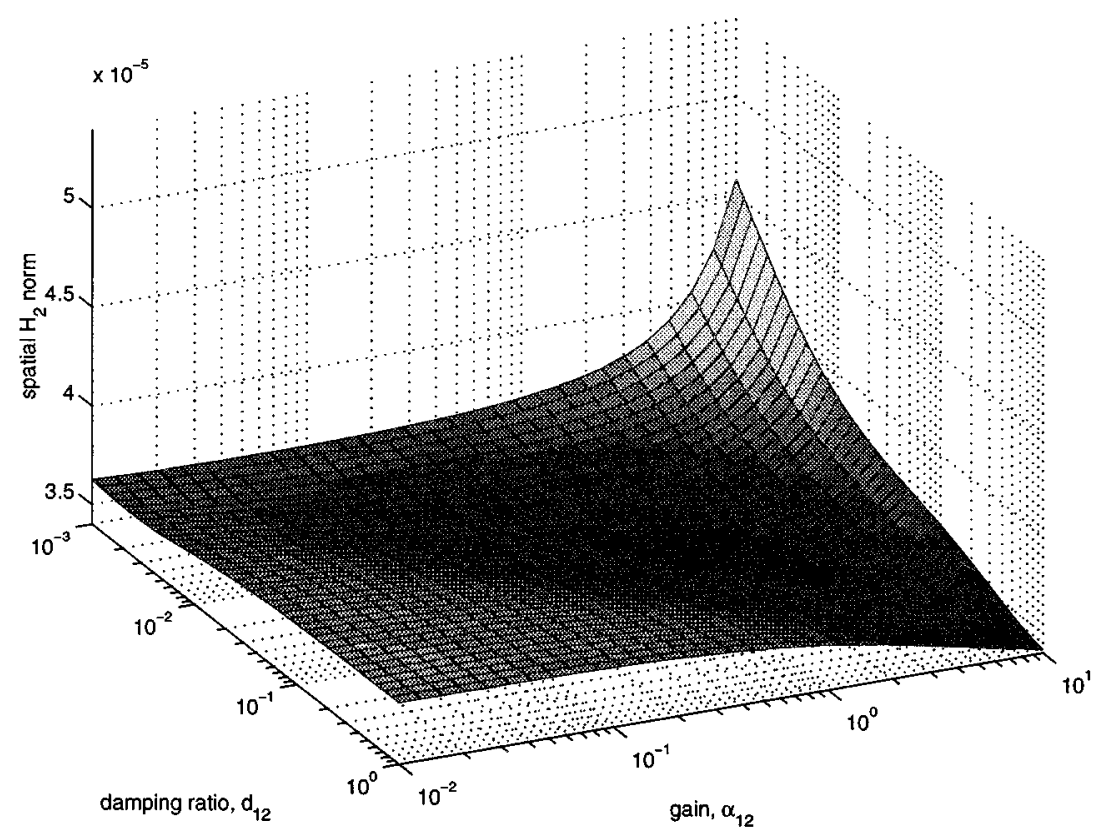

Fig. 5. Plot of cost function versus gain and damping ratio, mode 2 .

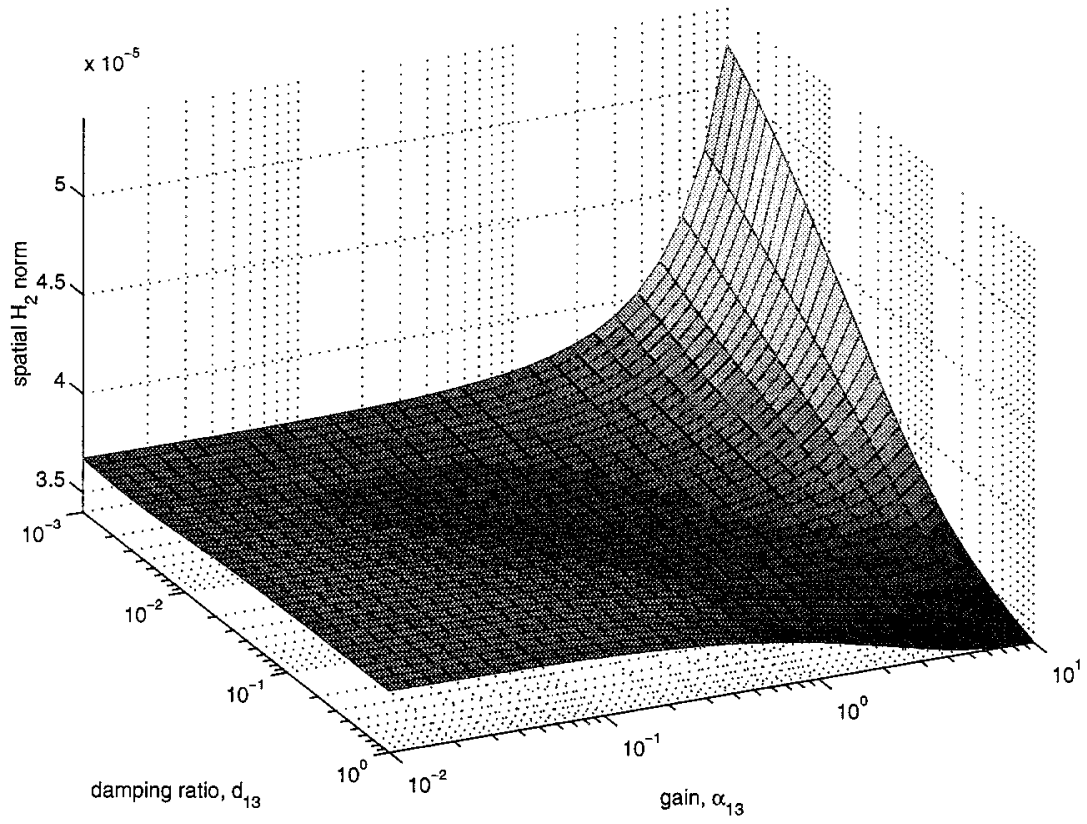

Fig. 6. Plot of cost function versus gain and damping ratio, mode 3 .

There is still a difficulty in using feedthrough terms in the system (52) since the spatial $\mathcal{H}_{2}$ norm of the system will not remain finite. To avoid this problem, we replace feedthrough terms with second-order out-of-bandwidth terms as suggested in [18]. Obviously, we have to ensure that the second order term has a zero-frequency content that is close to (52). The resonant frequency of the second-order system is set at $\omega_{c}=16 \mathrm{kHz}$, well above the bandwidth of interest of about $2 \mathrm{kHz}$. Also, a high damping ratio of $\zeta_{c}=0.7$ is used, so that the second-order system behaves like a low-pass filter. Since the controller and the system are highly resonant, this addition will have minimal effect on the optimization result. This will make the opti- mization procedure feasible, and will not increase the controller bandwidth. After the inclusion of the second-order mode to the system, the state-space model of the system in (34) can be replaced by the following system:

$$
\tilde{A}_{d}=\left[\begin{array}{cc}
0_{M+1 \times M+1} & I_{M+1 \times M+1} \\
\tilde{A}_{d 1_{M+1 \times M+1}} & \tilde{A}_{d 2_{M+1 \times M+1}}
\end{array}\right]
$$

where

$$
\begin{aligned}
& \tilde{A}_{d 1}=-\operatorname{diag}\left(\omega_{1}^{2}, \ldots, \omega_{M}^{2}, \omega_{c}^{2}\right) \\
& \tilde{A}_{d 2}=-2 \operatorname{diag}\left(\zeta_{1} \omega_{1}, \ldots, \zeta_{M} \omega_{M}, \zeta_{c} \omega_{c}\right)
\end{aligned}
$$




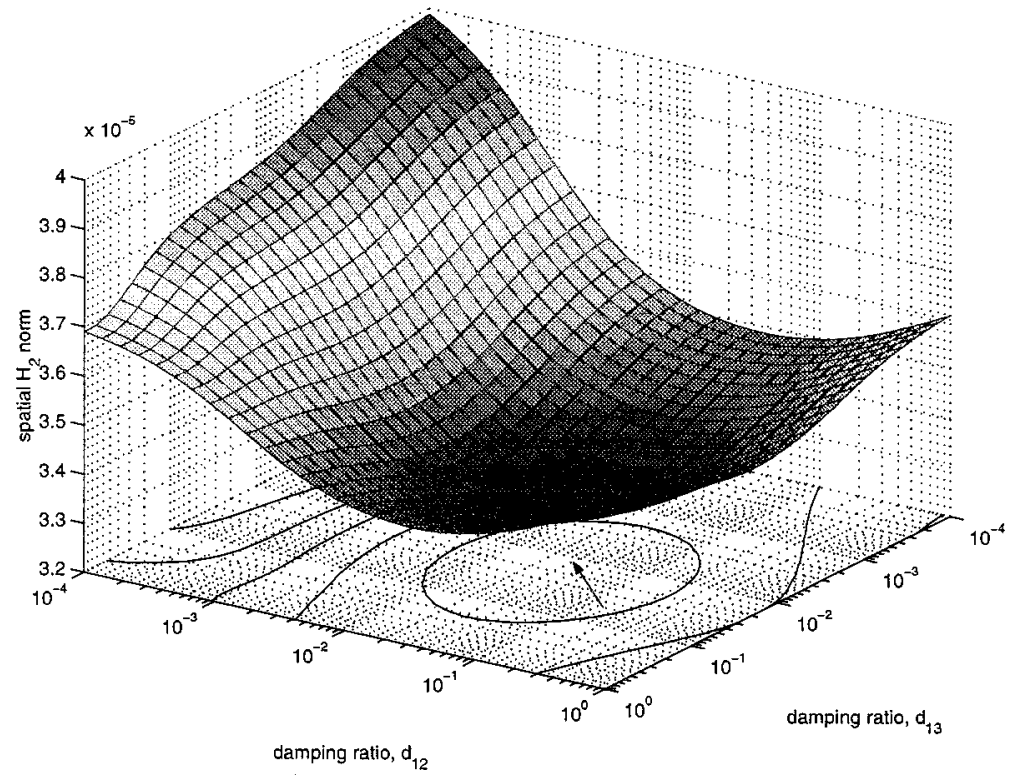

Fig. 7. Plot of cost function versus damping ratios.

and,

$$
\begin{aligned}
\tilde{B}_{d}= & {\left[0, \ldots, 0,0, P \Psi_{11}, \ldots, P \Psi_{M 1}, 1\right]^{T} } \\
\tilde{C}_{d}(x)= & {\left[W_{1}(x), \ldots, W_{M}(x), \omega_{c}^{2} \sum_{k=M+1}^{M_{\max }} K_{k}^{\mathrm{opt}} W_{k}(x),\right.} \\
& 0, \ldots, 0,0] \\
\tilde{C}_{V s}= & {\left[\Upsilon \Psi_{11}, \ldots, \Upsilon \Psi_{M 1}, D_{V s} \omega_{c}^{2}, 0, \ldots, 0,0\right] }
\end{aligned}
$$

where $\tilde{A}_{d}, \tilde{B}_{d}, \tilde{C}_{d}$, and $\tilde{C}_{V s}$ signify the corrected system of $A_{d}, B_{d}, C_{d}$, and $C_{V s}$ that are defined in (34).

Next, the closed-loop system as in (45) can be constructed from (37) and (55) and $\bar{\Gamma}$ can be calculated by incorporating the orthogonality property in (10)

$$
\bar{\Gamma}=\left[\begin{array}{cc}
\tilde{\Gamma}_{2 M+1 \times 2 M+1} & 0_{2 M+1 \times 2 \bar{I} N_{c}+1} \\
0_{2 \bar{I} N_{c}+1 \times 2 M+1} & 0_{2 \bar{I} N_{c}+1 \times 2 \bar{I} N_{c}+1}
\end{array}\right]
$$

where $\tilde{\Gamma}=\operatorname{diag}\left(1, \ldots, 1, \omega_{c}^{2}\left(\sum_{k=M+1}^{M_{\max }}\left(K_{k}^{\mathrm{opt}}\right)^{2}\right)^{1 / 2}\right)$.

One of the advantages of this particular controller structure is the ability to choose the resonant modes that need to be controlled. For this particular beam, the placement of the piezoelectric actuator-sensor pair on the beam (at $50 \mathrm{~mm}$ away from one end of the beam) results in relatively low control authority over the first resonant mode (at a frequency of about $20 \mathrm{~Hz}$ ). The low control authority of this mode is reflected in the frequency response (actuator voltage to sensor voltage) shown in Fig. 10 in Section VII. Thus, more control effort is needed to dampen the first mode than to control modes 2 and 3, for instance. In this experiment, we will demonstrate the controller's effectiveness in controlling some specific resonant modes. Here, we will attempt to control only the second and third resonant modes, and will leave the first mode uncontrolled.

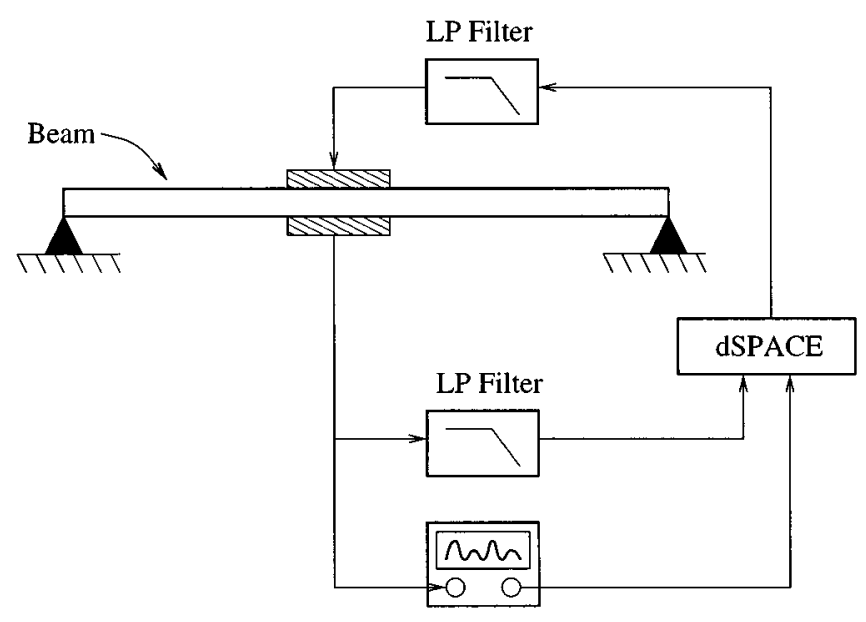

Signal Analyzer

Fig. 8. Experimental setup.

We choose a particular SISO resonant controller, i.e., $i=1$, of the following form:

$$
\begin{aligned}
K(s) & =\sum_{k=1}^{3}\left(\frac{\alpha_{i k} s\left(s+2 d_{i k} \omega_{k}\right)}{s^{2}+2 d_{i k} \omega_{k} s+\omega_{k}^{2}}-\alpha_{i k}\right) \\
& =\sum_{k=1}^{3} \frac{-\alpha_{i k} \omega_{k}^{2}}{s^{2}+2 d_{i k} \omega_{k} s+\omega_{k}^{2}}, \quad i=1, \quad \alpha_{11}=0 .
\end{aligned}
$$

Hence, we have achieved a particular resonant controller without any feedthrough term by choosing $R_{i k}=-\alpha_{i k}$.

To select the modal gains for our controller, we first need to consider the effect of these modal gains on the cost function (i.e., the spatial $\mathcal{H}_{2}$ norm of the closed-loop system). Consider the case when we wish to control each mode independently. A 

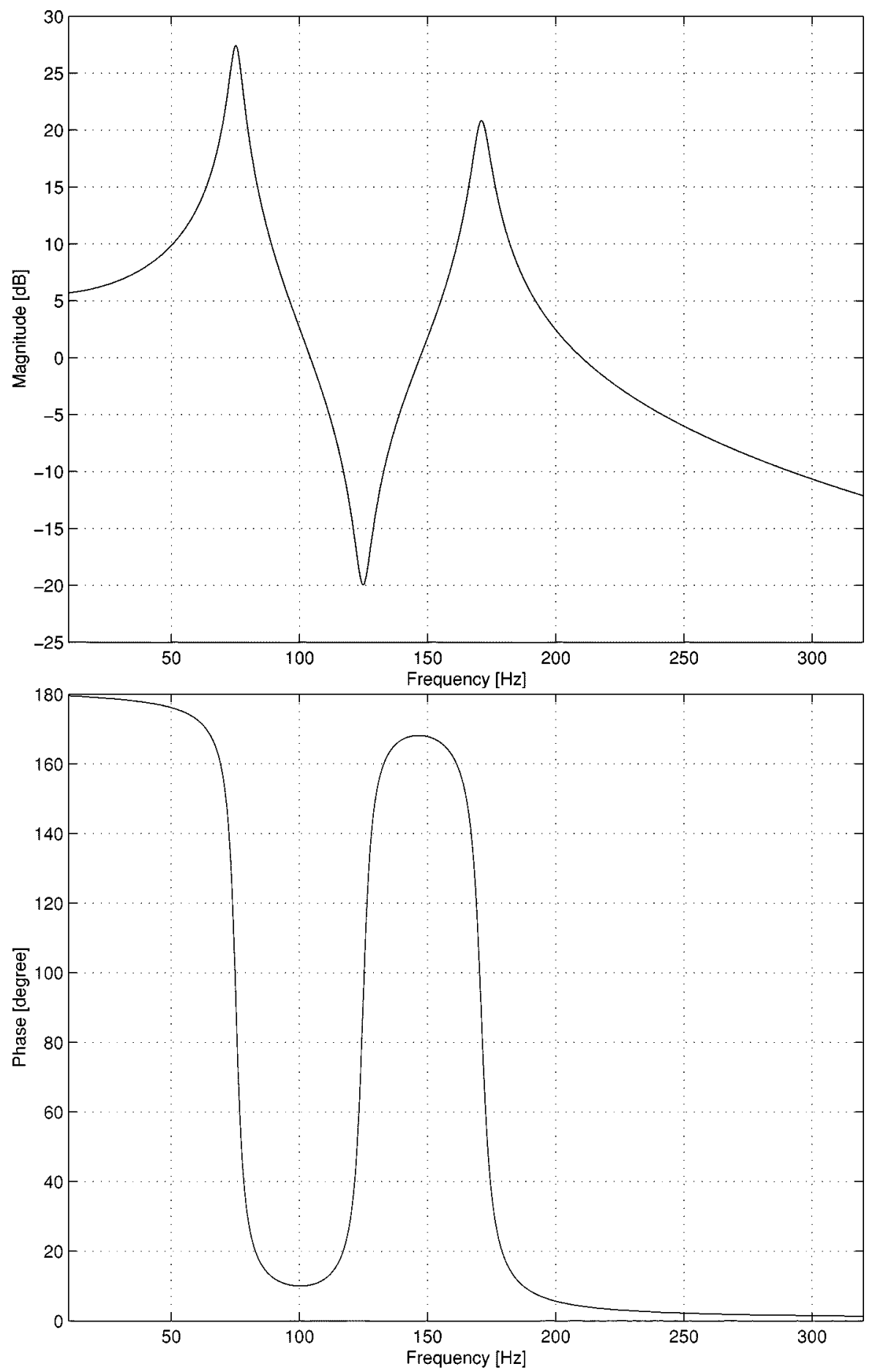

Fig. 9. Frequency response of the controller (input voltage to output voltage $[V / V]$ ).

plot of the cost function versus modal gain and damping ratio for each mode can be constructed. This would allow us to observe the effect of modal gain and damping ratio on our cost function. The plots for the second mode and the third mode are shown in Figs. 5 and 6.

Our objective here is to find a combination of modal gains, $\left\{\alpha_{i k}\right\}$, and damping ratios, $\left\{d_{i k}\right\}$, that would give a minimum cost function. From Figs. 5 and 6, it can be observed that the cost function becomes more warped as the modal gain increases. Hence, we may expect to achieve a smaller cost by increasing the modal gain and the damping ratio. Unfortunately, it turns out that this is not a desirable way of achieving our objective for vibration control. A very high-gain controller is not desirable because of excessive controller effort, sensitivity to noise, and reduction of system robustness. Furthermore, a high modal gain may result in the loss of highly localized nature of the controller.

Considering Figs. 5 and 6, we choose modal gains of $\alpha_{12}=$ 1.5 and $\alpha_{13}=0.4$ for the second and third modes, respectively. These gains give minimum cost function at damping ratios of $d_{12}=0.0330$ and $d_{13}=0.0209$ for the second and third modes. 

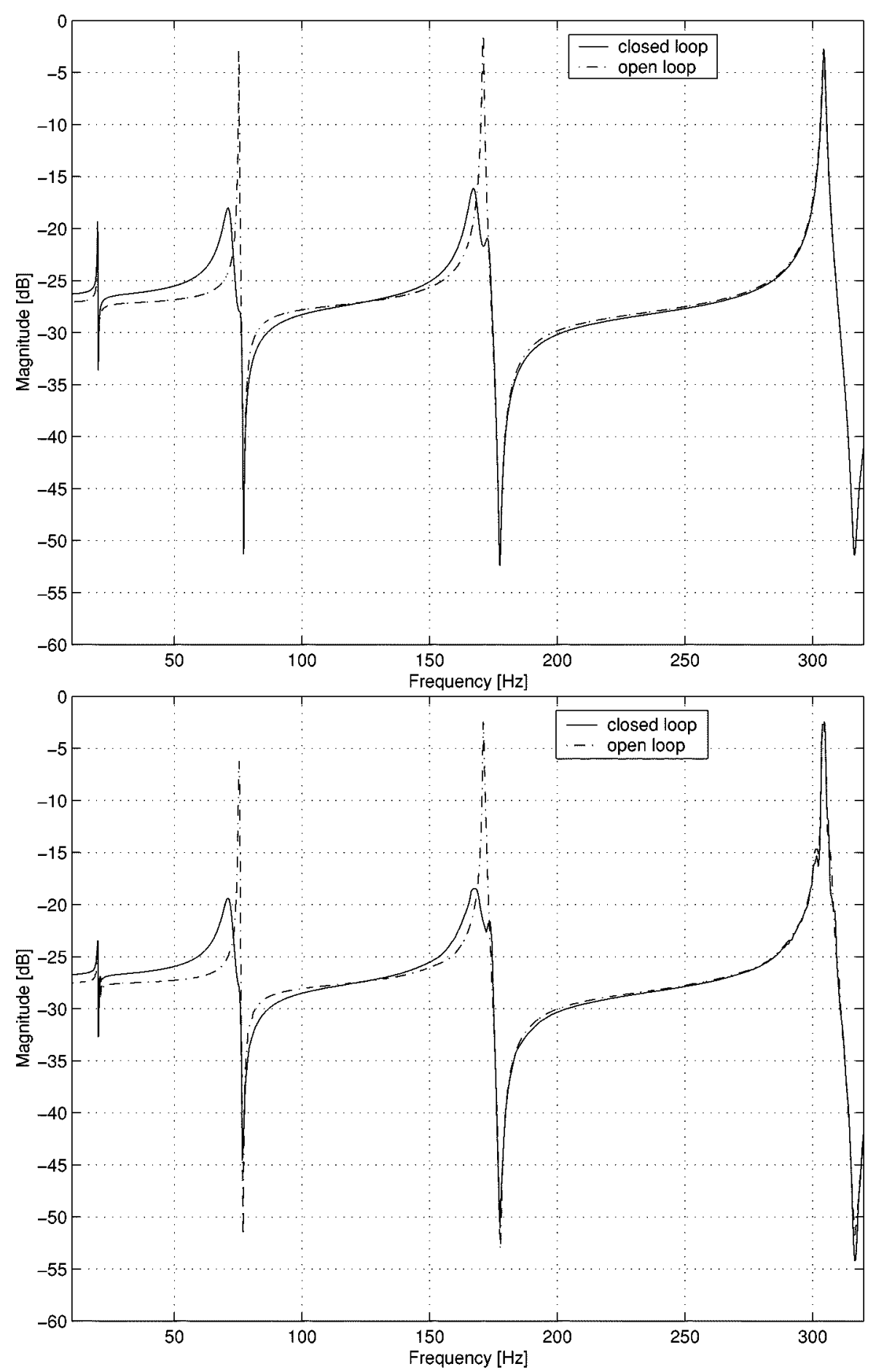

Fig. 10. Simulation and experimental frequency responses (actuator voltage to sensor voltage $[\mathrm{V} / \mathrm{V}]$ ).

If we choose larger modal gains that give lesser cost, the optimized damping ratios would be excessive.

Based on these parameters, single-mode controllers for modes 2 and 3 can be constructed. However, we wish to control the second and third modes simultaneously. In this exercise, modal gains of 1.5 and 0.4 are chosen for the second and third modes, respectively. These modal gains are based on the results of the previous single-mode optimization. This choice is reasonable since our controller has localized properties at resonances. The controller's gain at resonant mode 2 is not expected to influence the resonant mode 3 significantly, and vice versa.

The optimization is then performed using $d_{12}=0.0330$ and $d_{13}=0.0209$ as a starting point. A plot of the cost function versus the two damping ratios is shown in Fig. 7. The optimal damping ratios are $d_{12}=0.0320$ and $d_{13}=0.0182$ as shown by the arrow in Fig. 7. It is important to note that the damping ratios obtained here are relatively close to those obtained for single-mode controllers. This fact confirms that the effect of our resonant controller on the system is highly localized. Thus, our 

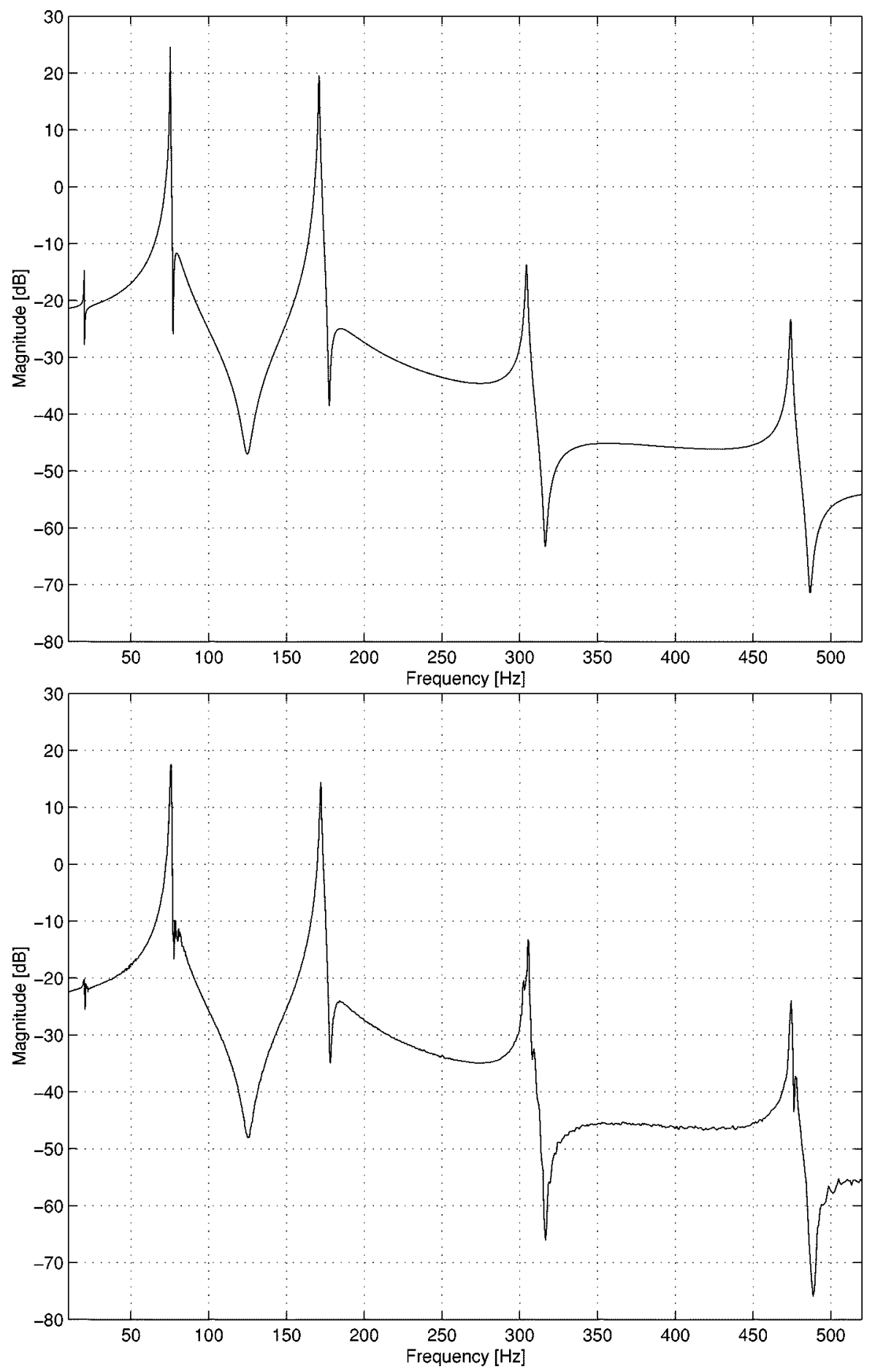

Fig. 11. Loop gain $[V / V]$ : simulation and experiment.

decision to use the modal gains obtained from a single-mode control is reasonable.

\section{EXPERIMENTAL RESULTS}

The experiment was set at the Laboratory for Dynamics and Control of Smart Structures at the University of Newcastle, Australia. The experimental setup is depicted in Fig. 8. The controller was implemented on a dSPACE DS1103 rapid prototyping Controller Board using Matlab and Simulink software.
The sampling frequency was set at $20 \mathrm{KHz}$. The cutoff frequencies of the two low-pass filters were set at $10 \mathrm{KHz}$ each. An HP89410A Dynamic Signal Analyzer was used to obtain frequency responses from the piezoelectric laminate beam. A Polytec PSV-300 Laser Doppler Scanning Vibrometer was also used to obtain the frequency response of the beam's vibration. This laser vibrometer allows accurate vibration measurement at any point on the beam by measuring the Doppler frequency shift of the laser beam that is reflected back from the vibrating beam. Important parameters of the beam, such as resonant frequencies 

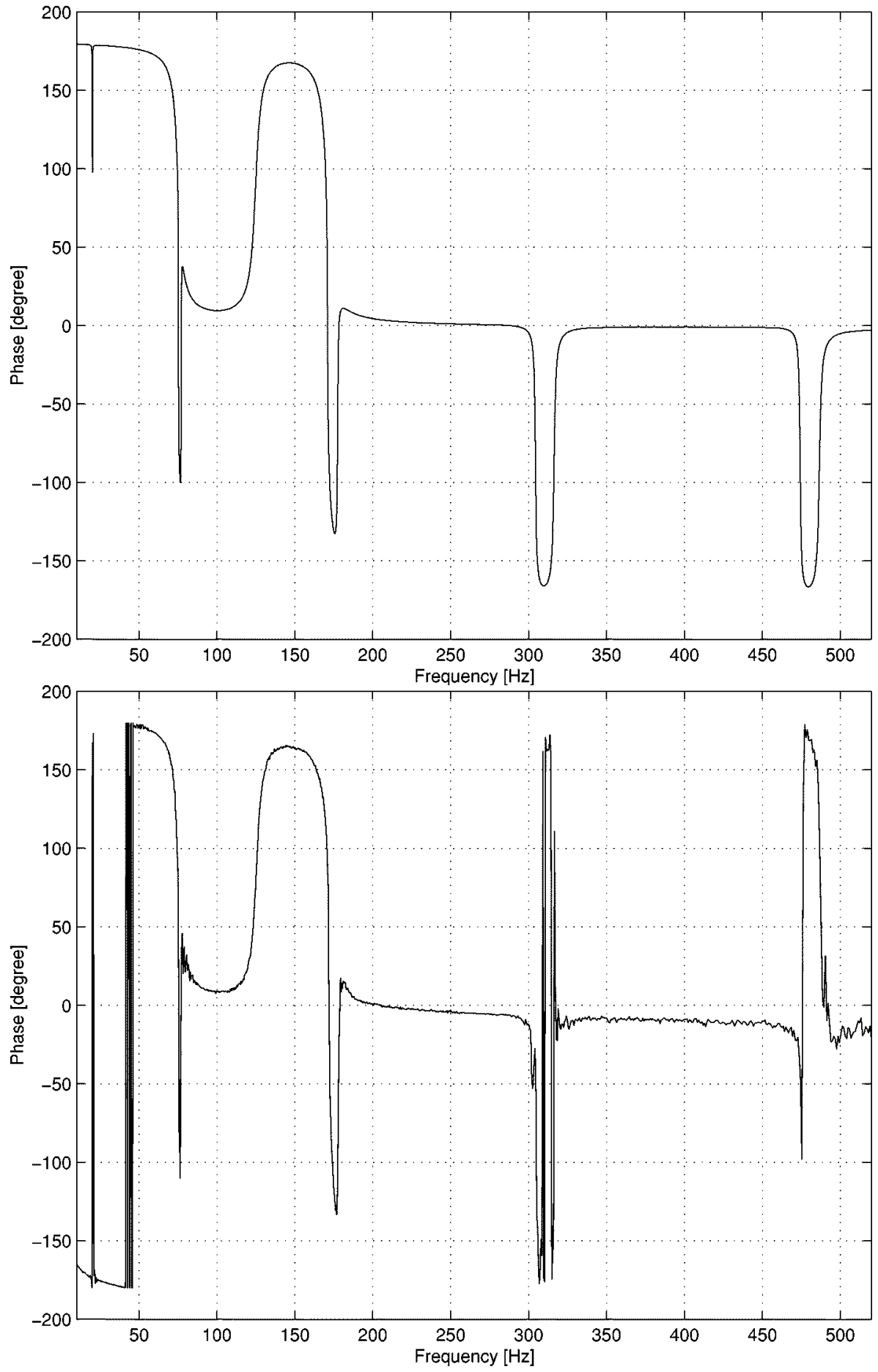

Fig. 11. (Continued.) Loop gain $[V / V]$ : simulation and experiment.

and damping ratios, were obtained from the experimental apparatus and were used to correct our model.

Our simulation and experimental results are presented as follows. The frequency response of the controller is plotted in Fig. 9. It can be observed that the controller has a resonant structure, as expected. Fig. 10 shows a comparison of the frequency responses (actuator voltage to sensor voltage) of the open-loop and closed-loop systems. Simulation and experimental results are presented. It can be observed that the performance of the controller applied to the real system is as predicted by the model. The resonant responses of modes 2 and 3 of the system have been reduced significantly. It can also be observed that the controller has minimal effect on modes 1 and 4.

Fig. 11 shows the experimental and simulated loop gain up to $520 \mathrm{~Hz}$. Our simulation gives a theoretical value of infinity for the gain margin and a phase margin of $-35.9^{\circ}$ at $72.1 \mathrm{~Hz}$. An infinite margin in this case is a direct consequence of the passivity of the closed-loop system as explained in Section IV. For a SISO system, this means that, theoretically, the Nyquist 




Fig. 12. Simulation spatial frequency response: actuator voltage—beam deflection $[m / V]$ (open loop).

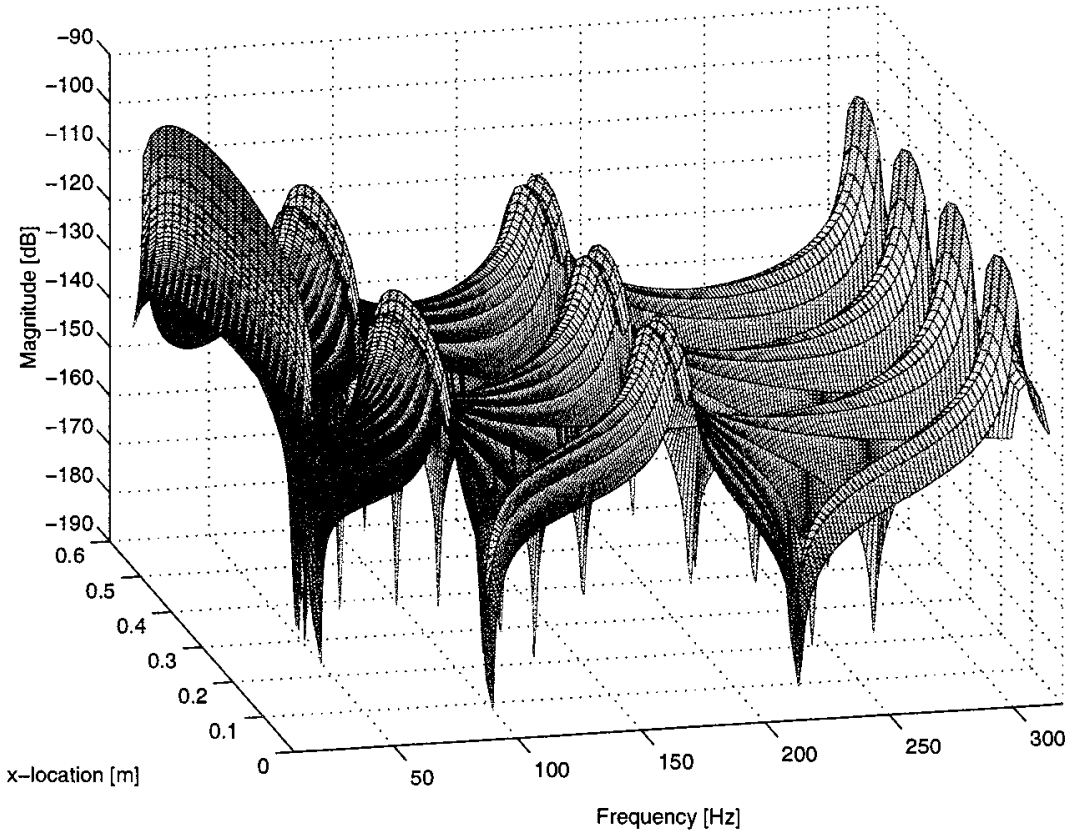

Fig. 13. Simulation spatial frequency response: actuator voltage—beam deflection $[\mathrm{m} / \mathrm{V}]$ (closed loop).

path never crosses the negative real axis. The experiment gives a gain margin of $19.5 \mathrm{~dB}$ at $41.7 \mathrm{~Hz}$, and a phase margin of $-41.9^{\circ}$ at $72.6 \mathrm{~Hz}$. These results demonstrate a sufficient robustness for our controller.

Our controller was designed to minimize the spatial $\mathcal{H}_{2}$ norm of the closed-loop system. To show the controller effect on the frequency response of deflection over the entire beam, we have also plotted the spatial frequency responses of the beam.

Figs. 12 and 13 compare the spatial frequency responses of the uncontrolled and controlled beam using the simulation results. The location $x$ is measured from one end of the beam, which is closer to the patches, while the frequency response is in terms of the beam's transverse deflection (deflection in $Z$-axis, see Fig. 1). The resonant responses of modes 2 and 3 have been reduced because of the controller action.

Next, a Polytec PSV-300 Laser Scanning Vibrometer was used to obtain the experimental frequency response of the beam's vibration at a number of points along the beam. The results allow us to plot the spatial frequency responses of the uncontrolled and controlled beam from the experiments as shown in Figs. 14 and 15, respectively. Our experiment confirms the simulation results, where we obtain vibration reduction for modes 2 and 3 over the entire structure. The experiments show that the resonant responses of modes 2 and 


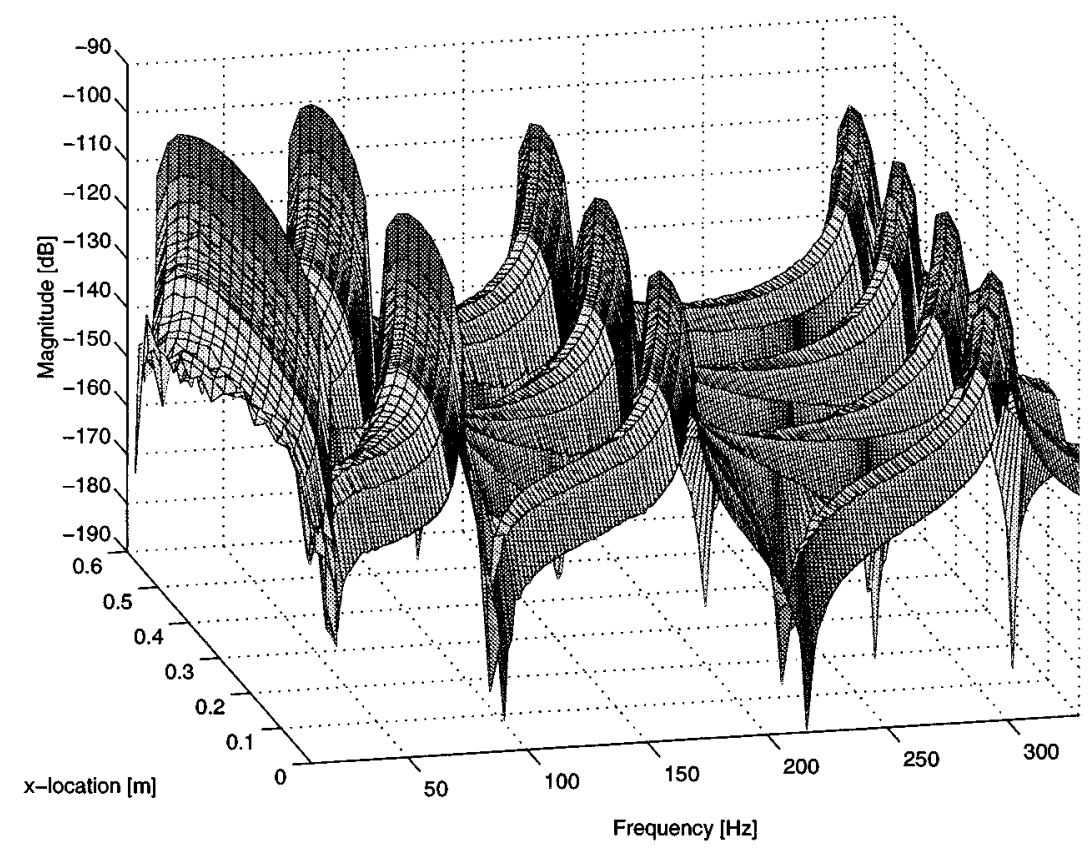

Fig. 14. Experimental spatial frequency response: actuator voltage—beam deflection $[\mathrm{m} / \mathrm{V}]$ (open loop).

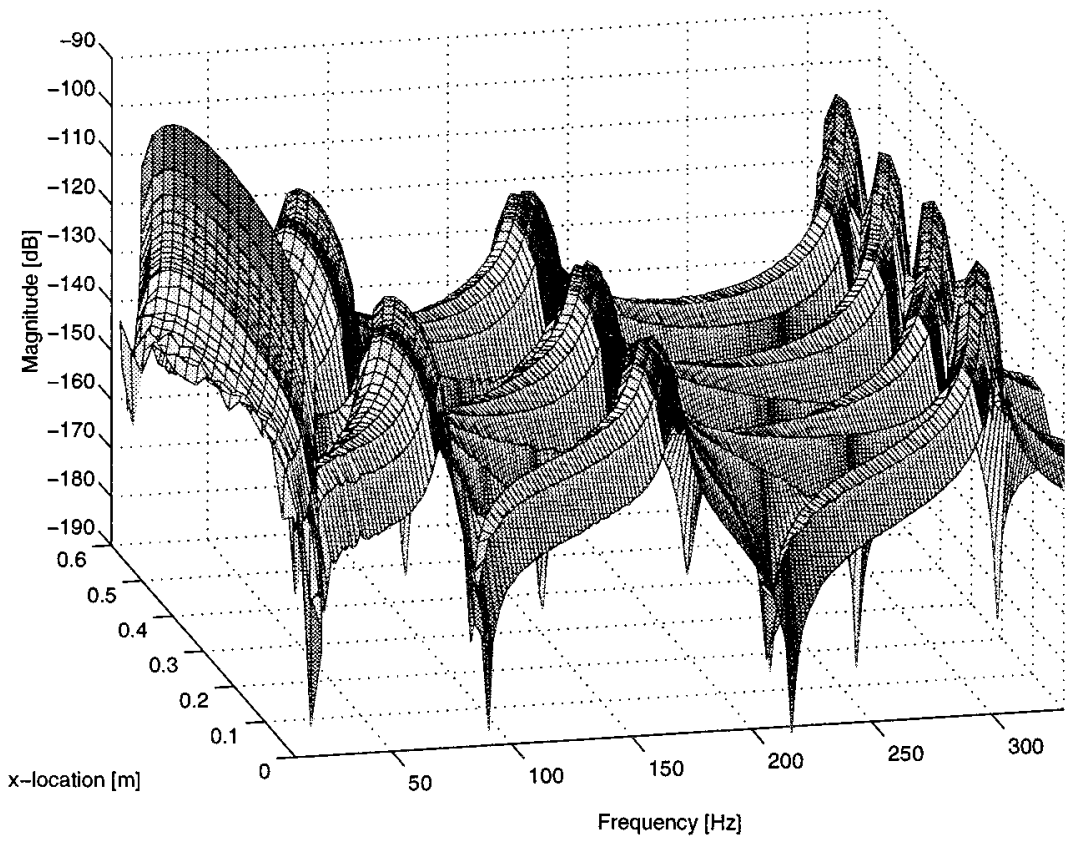

Fig. 15. Experimental spatial frequency response: actuator voltage—beam deflection $[\mathrm{m} / \mathrm{V}]$ (closed loop).

3 have been reduced by around 20 and $15 \mathrm{~dB}$ respectively, over the entire beam.

\section{CONCLUSION}

A class of resonant controllers has been introduced in this paper. These controllers can be applied to structures which contain compatible pairs of collocated actuators and sensors. The controller reduces resonant responses of the structure by increasing the system damping at those resonant frequencies. Passivity of the system guarantees closed-loop stability in the presence of uncontrolled high-frequency modes and inaccurate structural models. The controller damping factors are chosen such that the spatial $\mathcal{H}_{2}$ norm of the closed-loop system is minimized. It is observed that such a controller results in suppression of the transverse deflection of the entire structure. The experiment presented shows the effectiveness of the developed controller in reducing the structural vibrations on a piezoelectric laminate beam.

\section{REFERENCES}

[1] H. R. Pota, S. O. R. Moheimani, and M. Smith, "Resonant controllers for flexible structures," in Proc. 38th IEEE Conf. Decision Contr., Phoenix, AZ, Dec. 1999, pp. 631-636. 
[2] H. S. Tzou, "Integrated distributed sensing and active vibration suppression of flexible manipulators using distributed piezoelectrics," J Robot. Syst., vol. 6, no. 6, pp. 745-767, 1989.

[3] C. K. Lee, "Piezoelectric Laminates for Torsional and Bending Modal Control: Theory and Experiment," Ph.D. dissertation, Cornell Univ., Ithaca, NY, 1987.

[4] C. K. Lee and F. C. Moon, "Modal sensors/actuators," ASME J. Appl. Mechanics, vol. 57, pp. 434-441, June 1990.

[5] S. O. R. Moheimani and T. Ryall, "Considerations in placement of piezoceramic actuators that are used in structural vibration control," in Proc. 38th IEEE Conf. Decision Contr., Phoenix, AZ, Dec. 1999, pp. $1118-1123$.

[6] R. L. Clark, W. R. Saunders, and G. P. Gibbs, Adaptive Structures Dynamics and Control. New York: Wiley, 1998.

[7] E. K. Dimitriadis, C. R. Fuller, and C. A. Rogers, "Piezoelectric actuators for distributed vibration excitation of thin plates," ASME J. Vibr. Acoust., vol. 113, pp. 100-107, Jan. 1991.

[8] C. R. Fuller, S. J. Elliot, and P. A. Nelson, Active Control of Vibration. New York: Academic, 1996.

[9] H. T. Banks, R. C. Smith, and Y. Wang, Smart Material Structures: Modeling, Estimation and Control. New York: Wiley, 1996.

[10] H. R. Pota and T. E. Alberts, "Multivariable transfer functions for a slewing piezoelectric laminate beam," ASME J. Dyn. Syst., Measurement, Contr., vol. 117, pp. 352-359, Sept. 1995.

[11] M. J. Balas, "Active control of flexible systems," J. Optim. Theory Applicat., vol. 25 , no. 3, pp. 415-436, 1978.

[12] — "Feedback control of flexible systems," IEEE Trans. Automat. Contr., vol. AC-23, 1978.

[13] S. O. R. Moheimani and M. Fu, "Spatial $\mathcal{H}_{2}$ norm of flexible structures and its application in model order selection," in Proc. 37th IEEE Conf Decision Contr., Tampa, FL, Dec. 1998, pp. 3623-3624.

[14] L. Meirovitch, Elements of Vibration Analysis. New York: McGrawHill, 1975.

[15] H. Kwakernaak and R. Sivan, Modern Signals and Systems. Englewood Cliffs, N.J.: Prentice-Hall, 1991.

[16] P. C. Hughes, "Space structure vibration modes: How many exist? Which ones are important?," IEEE Contr. Syst. Mag., pp. 22-28, Feb. 1987.

[17] S. M. Joshi and S. Gupta, "On a class of marginally stable positive-real systems," IEEE Trans. Automat. Contr., vol. 41, pp. 152-155, Jan. 1996

[18] R. L. Clark, "Accounting for out-of-bandwidth modes in the assumed modes approach: Implications on colocated output feedback control," Trans. ASME, vol. 119, pp. 390-395, Sept. 1997.
[19] S. O. R. Moheimani, "Minimizing the effect of out-of-bandwidth dynamics in the models of reverberant systems that arise in modal analysis: Implications on spatial $\mathcal{H}_{\infty}$ control," Automatica, vol. 36, pp. 1023-1031, 2000

[20] — "Minimizing the effect of out of bandwidth modes in truncated structure models," ASME J. Dyn. Syst., Measurement, Contr., vol. 122, pp. 237-239, Mar. 2000.

[21] - "Experimental verification of the corrected transfer function of a piezolectric laminate beam," IEEE Trans. Contr. Syst. Technol., vol. 8, pp. 660-666, July 2000.

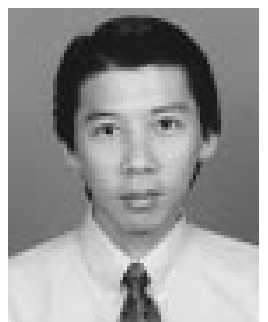

Dunant Halim was born in Indonesia in 1974. He received the B.Eng. degree (first class honors) in aerospace engineering from the Royal Melbourne Institute of Technology, Victoria, Australia, in 1999. $\mathrm{He}$ is currently pursuing the Ph.D. degree in the Department of Electrical and Computer Engineering at the University of Newcastle, NSW, Australia.

His current research is in vibration control of smart structures using piezoelectric devices.

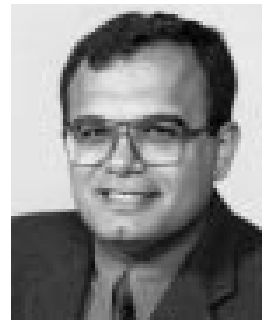

S. O. Reza Moheimani (M'97) was born in Shiraz, Iran, in 1967. He received the B.Sc. degree from Shiraz University, Iran, in 1990 and the M.Eng.Sc. and $\mathrm{Ph} . \mathrm{D}$. degrees from the University of New South Wales, Australia, in 1993 and 1996, respectively, all in electrical and electronic engineering.

In 1996, he was a Postdoctoral Research Fellow at the School of Electrical and Electronic Engineering, Australian Defence Force Academy, Canberra. In 1997, he joined the Department of Electrical and Computer Engineering at the University of Newcastle, where he is currently a Senior Lecturer. He is a member of the Centre for Integrated Dynamics and Control, an Australian Government Special Research Centre. His interests include robust control and filtering, active control of noise and vibrations, smart structures, and signal processing. 